Article

\title{
An Improved Imaging Algorithm for Multi-Receiver SAS System with Wide-Bandwidth Signal
}

\author{
Xuebo Zhang ${ }^{1, *(\mathbb{D})}$ and Peixuan Yang ${ }^{2}$ \\ 1 College of Physics and Electronic Engineering, Northwest Normal University, Lanzhou 730070, China \\ 2 Acoustic Signal \& Electronics Science and Technology Corporation, Lanzhou 730050, China; \\ peixuan_yang@126.com \\ * Correspondence: xuebo_zhang@sina.cn
}

Citation: Zhang, X.; Yang, P. An Improved Imaging Algorithm for Multi-Receiver SAS System with Wide-Bandwidth Signal. Remote Sens. 2021, 13, 5008. https://doi.org/ $10.3390 /$ rs13245008

Academic Editor: Andrzej Stateczny

Received: 6 November 2021

Accepted: 8 December 2021

Published: 9 December 2021

Publisher's Note: MDPI stays neutral with regard to jurisdictional claims in published maps and institutional affiliations.

Copyright: (c) 2021 by the authors. Licensee MDPI, Basel, Switzerland. This article is an open access article distributed under the terms and conditions of the Creative Commons Attribution (CC BY) license (https:/ / creativecommons.org/licenses/by/ $4.0 /)$.
Abstract: When the multi-receiver synthetic aperture sonar (SAS) works with a wide-bandwidth signal, the performance of the range-Doppler (R-D) algorithm is seriously affected by two approximation errors, i.e., point target reference spectrum (PTRS) error and residual quadratic coupling error. The former is generated by approximating the PTRS with the second-order term in terms of the instantaneous frequency. The latter is caused by neglecting the cross-track variance of secondary range compression (SRC). In order to improve the imaging performance in the case of wide-bandwidth signals, an improved R-D algorithm is proposed in this paper. With our method, the multi-receiver SAS data is first preprocessed based on the phase center approximation (PCA) method, and the monostatic equivalent data are obtained. Then several sub-blocks are generated in the cross-track dimension. Within each sub-block, the PTRS error and residual quadratic coupling error based on the center range of each sub-block are compensated. After this operation, all sub-blocks are coerced into a new signal, which is free of both approximation errors. Consequently, this new data is used as the input of the traditional R-D algorithm. The processing results of simulated data and real data show that the traditional R-D algorithm is just suitable for an SAS system with a narrow-bandwidth signal. The imaging performance would be seriously distorted when it is applied to an SAS system with a wide-bandwidth signal. Based on the presented method, the SAS data in both cases can be well processed. The imaging performance of the presented method is nearly identical to that of the back-projection (BP) algorithm.

Keywords: synthetic aperture sonar; multi-receiver; R-D algorithm; approximation error; imaging performance

\section{Introduction}

Originating from synthetic aperture radar (SAR) [1-10], synthetic aperture sonar (SAS) [11-16] attracts investigators' interests due to its high resolution in the underwater field. Nowadays, it is widely applied to underwater mapping $[14,15,17,18]$, target recognition [19-22], and so on. Considering the traditional monostatic SAS system, the maximum imaged swath is determined by the pulse repetition frequency (PRF), and the low PRF allows for a wide swath. At the same time, the sampling of the along-track signal is conducted by this PRF. According to the sampling theorem, the PRF should be greater or equal to the Doppler bandwidth. When a too low PRF is exploited, there would be ambiguous targets in the SAS image as the along-track sampling with this low PFR does not satisfy the Nyquist-Shannon theorem [23]. Generally speaking, the SAS system parameters are subject to a trade-off, as the along-track resolution is only improved at the cost of a decreased swath or vice versa. The inherent limitation of monostatic SAS between along-track resolution and cross-track swath demands the new SAS systems. One of the promising systems is the multi-receiver SAS, which employs a single transmitting element and a receiver array. This system can successfully overcome the limitations of 
the monostatic SAS system. With the multi-receiver SAS system, the echo signal can be sampled by multiple receiver elements [24-26]. Consequently, the effective sampling rate of the along-track signal is increased while the transmitting PRF remains unaltered.

The high-resolution imagery introduced by the multi-receiver SAS system is obtained at the cost of increased complexity with respect to the processing of the multi-receiver SAS raw data. With monostatic SAS, the point target reference spectrum (PTRS) can be easily obtained based on the method of the stationary phase [27]. However, the multi-receiver SAS system has many bistatic SAS systems [28,29]. Considering each bistatic SAS, the transmitter and receiver individually contribute to the two-way slant range. That is to say, the two-way slant range has double square rooted terms [30]. Due to this reason, the point of the stationary phase (PSP) and PTRS cannot be analytically deduced based on the method of the stationary phase. To solve this problem, the phase center approximation (PCA) method [31-36] is used. With this method, a virtual element located midway between the transmitter and receiver transmits and receives the signal, and the two-way slant range with double square rooted terms can be coerced into a slant range with a single square rooted term. In [31], the two-way slant range is directly approximated by the round-trip range between the virtual element and target. This operation neglects the PCA error introduced by the accurate two-way slant range and approximate range. When the distance between the transmitter and receiver is large, this operation leads to large errors. In [32], the PCA error is considered. Furthermore, they also consider the stop-and-hop error [37]. However, the stop-and-hop error is compensated for using the reference range from the transmitter to scene center. That is to say, the targets at the edge of the mapping swath would suffer from a large stop-and-hop error. Furthermore, the PCA error and stop-and-hop error are separately considered, and the coupling between both errors is neglected. In [33], the PCA error is analytically deduced based on the imaging geometry. Unfortunately, the stop-and-hop error is not compensated. The distributed target would be distorted when the SAS system works with a wide swath. In [34], the PCA idea is used two times. One is to obtain the phase center between transmitter and receiver under the case of the stop-and-hop assumption, and the other is to get the phase center between transmitter and receiver without the stop-and-hop assumption. After deducing the approximation error between the accurate range and approximated one, the PCA error and stop-and-hop error are deduced with a complicated expression. Bellettinis' PCA error is directly used in [35], where Pailhas et al. discuss the impact of temporal Doppler on SAS imagery. With their work, the temporal Doppler effect introduced by the sonar motion during signal transmission and reception is further discussed. However, the spatial Doppler introduced by sonar motion during signal transmission and reception is not considered. In this paper, the PCA method is still used. Compared to the method in [33], the stop-and-hop error, together with the coupling between PCA error and stop-and-hop error, is considered in this paper. Compared to the method in [34], we simplify the deduction of PCA error and stop-and-hop error based on second-order Taylor expansion and inverse Taylor expansion. Moreover, the presented model in this paper has a much better understanding. Compared to the method in [35], we mainly consider the spatial Doppler introduced by stop-and-hop error and neglect the temporal Doppler effect, as it is not a dominant factor influencing the SAS imaging performance based on the experiment results in [35]. For clarity, the PCA error is called the signal model error [36] in this paper.

With the analytical PTRS, the range-Doppler (R-D) algorithm [27,38] cannot be directly developed yet. Based on the analytical PTRS, Taylor expansion should be further exploited. For traditional R-D algorithms, the PTRS should be individually expanded up to the first-order term [39] and the second-order term [40,41] with respect to the instantaneous frequency. We find that the Taylor expansion of PTRS would also result in error, as the higher-order terms are all neglected. For clarity, we call it the PTRS error. The firstorder term denotes the range cell migration (RCM) or the linear coupling between crosstrack and along-track dimensions [27]. The interpolator is often exploited to perform the $\mathrm{RCM}$ correction (RCMC). The second-order term denotes the quadratic coupling between 
cross-track and along-track dimensions [27]. It is equivalent to a chirp modulation in the cross-track dimension. However, the equivalent chirp rate highly depends on the range, instantaneous frequency, and Doppler frequency. That is to say, the equivalent chirp rate is a cross-track variant, and the cross-track variance is hard to compensate in the two-dimensional (2-D) frequency domain. For simplicity, the equivalent chirp rate at the reference range is often exploited when users conduct the R-D algorithm. With this operation, it is easily concluded that the targets not located at the reference range would suffer from residual quadratic coupling error, which would further affect the SAS focusing performance.

In general, the multi-receiver SAS R-D algorithm [38] would suffer from three approximation errors, i.e., signal model error, PTRS error, and residual quadratic coupling error. The traditional R-D algorithm [38] just pays attention to the signal model error. In other words, the other two approximation errors are not focused on by users. They would seriously affect the SAS imaging performance in the case of wide-bandwidth and wide swath SAS systems. In this paper, we quantitatively discuss these three errors. Then, an improved R-D algorithm considering the compensation of PTRS error and residual quadratic coupling error is presented based on the sub-block processing method. With the presented method, the higher-order phase errors can be well compensated. Consequently, the high-performance results, which are close to the imaging performance of the back projection (BP) algorithm [42-44], can be generated. The simulations and real data processing further validate our method.

This paper is organized as follows. Section 2 introduces the multi-receiver imaging geometry and PTRS. In Section 3, the signal model error, PTRS error, and residual quadratic coupling error are quantitatively discussed in detail. Section 4 discusses an improved R-D algorithm that can be applied to a wide-bandwidth signal-based multi-receiver SAS system. In Section 5, simulated and real datasets are used to validate the presented method. The last section summarizes this paper.

\section{Imaging Geometry and PTRS}

\subsection{Imaging Geometry}

The along-track aperture of the element and that of the adjacent element phase center are $d . M$ receivers are uniformly placed in the along-track dimension, which is also called the azimuth dimension. The imaging geometry of the multi-receiver SAS system is shown in Figure 1. The horizontal axis is the cross-track dimension, which is also named the range dimension. The black rectangle is used as the transmitting element. The other rectangles are considered to be receivers, which are able to cover the illuminated area.

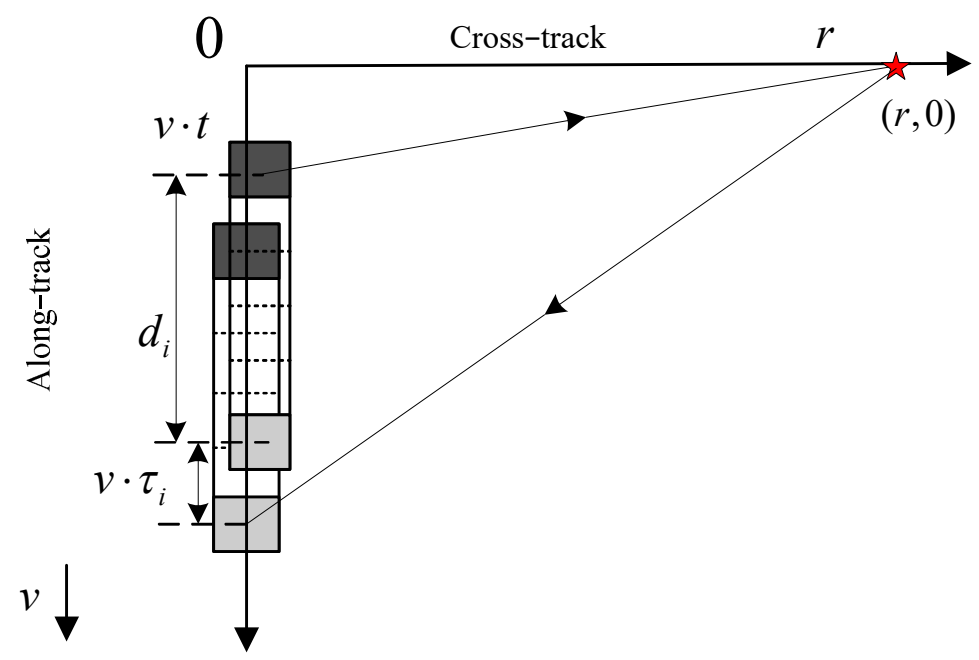

Figure 1. Imaging geometry of the multi-receiver SAS. 
Assuming that an ideal point target is a swath, and the minimal slant range between the target and sonar is $r$, the received echo of the $i$-th receiver is given by:

$$
s s_{i}(\tau, t)=p\left(\tau-\frac{R_{i}(t ; r)}{c}\right) \omega_{a}(t) \cdot \exp \left\{-\mathrm{j} 2 \pi f_{c} \frac{R_{i}(t ; r)}{c}\right\},
$$

where $R_{i}(t ; r)=\sqrt{r^{2}+(v t)^{2}}+\sqrt{r^{2}+\left(v t+d_{i}+v \tau_{i}\right)^{2}}$ denotes the two-way slant range. $v$ represents the sonar velocity. $\tau_{i}$ is the accurate delay-time of the transmitted signal [37]. $\omega_{a}(t)$ is the two-way pattern of elements. $c$ is the sound speed in the water. $f_{c}$ is the carrier frequency. $d_{i}=i d(i \in[1, M])$ is the distance between the transmitter and the $i$-th receiver. $p(\tau)$ stands for the chirp signal. $\tau$ and $t$ denote the fast time and slow time corresponding to the cross-track and along-track dimensions, respectively.

\subsection{PTRS}

For multi-receiver SAS echo sampling, the input is the target reflectivity, and the output is the echo signal. Therefore, the SAS imagery is an inverse process of echo sampling. By designing the filter function based on the PTRS, the targets can be reconstructed. At this point, it can be found that the PTRS plays an important role in SAS imagery. Applying Fourier transformation (FT) to (1) in the cross-track dimension yields:

$$
S s_{i}\left(f_{\tau}, t\right)=P\left(f_{\tau}\right) \omega_{a}(t) \cdot \exp \left\{-\mathrm{j} 2 \pi\left(f_{c}+f_{\tau}\right) \frac{R_{i}(t ; r)}{c}\right\},
$$

where $f_{\tau}$ is the instantaneous frequency corresponding to the fast time.

The PTRS can be obtained by transforming (2) into a 2-D frequency domain. Based on along-track FT, (2) is further reformulated as:

$$
S S_{i}\left(f_{\tau}, f_{t}\right)=P\left(f_{\tau}\right) \omega_{a}(t) \cdot \int_{-T_{s} / 2}^{T_{s} / 2} \exp \left\{-\mathrm{j} 2 \pi\left(f_{c}+f_{\tau}\right) \frac{R_{i}(t ; r)}{c}-\mathrm{j} 2 \pi f_{t} t\right\} d t,
$$

where $f_{t}$ represents the Doppler frequency corresponding to slow time in the along-track dimension. $T_{S}$ is the synthetic aperture time.

The exponential phase in (3) is expressed as:

$$
\varphi_{i}\left(f_{\tau}, f_{t} ; r\right)=-2 \pi\left(f_{c}+f_{\tau}\right) \frac{R_{i}(t ; r)}{c}-2 \pi f_{t} t,
$$

Based on the method of stationary phase [27], the integral phase is expressed as:

$$
\frac{\partial R_{i}\left(t_{i} ; r\right)}{\partial t}+\frac{c f_{t}}{f_{c}+f_{\tau}}=0
$$

where $t_{i}$ is the PSP. Substituting $t_{i}$ into (4) yields the PTRS phase, which is:

$$
\varphi_{i}\left(f_{\tau}, f_{t} ; r\right)=-2 \pi\left(f_{c}+f_{\tau}\right) \frac{R_{i}\left(t_{i} ; r\right)}{c}-2 \pi f_{t} t_{i}
$$

Since the two-way slant range $R_{i}(t ; r)=\sqrt{r^{2}+(v t)^{2}}+\sqrt{r^{2}+\left(v t+d_{i}+v \tau_{i}\right)^{2}}$ possesses double square rooted terms, there is no analytical solution to (5). To solve this problem, the two-way slant range is expanded up to the second-order term, and it is given by: 


$$
\begin{aligned}
R_{i}(t ; r) & =\sqrt{r^{2}+(v t)^{2}}+\sqrt{r^{2}+\left(v t+d_{i}+v \tau_{i}\right)^{2}} \\
& \approx \underbrace{\sqrt{r^{2}+(v t)^{2}}+\sqrt{r^{2}+\left(v t+d_{i}+v \frac{2 r}{c}\right)^{2}}}_{\Downarrow \text { Taylor expansion }} \\
\approx & \underbrace{2 r+\frac{v^{2}}{r}\left[t+\left(\frac{r}{c}+\frac{d_{i}}{2 v}\right)\right]^{2}+\frac{1}{4 r}\left(v \frac{2 r}{c}+d_{i}\right)^{2}}_{\Downarrow \text { Inverse Taylor expansion }} \\
& \approx 2 \sqrt{r^{2}+\left(v t+v \frac{r}{c}+\frac{d_{i}}{2}\right)^{2}}+\frac{\left(v \frac{2 r}{c}+d_{i}\right)^{2}}{4 r}
\end{aligned}
$$

In (7), an approximation $\tau_{i} \approx 2 r / c$ is used to simplify the deducing. Substituting (7) into (5) yields PSP, which is:

$$
t_{i}=-\frac{c r f_{t}}{v\left(f_{c}+f_{\tau}\right)} \cdot \frac{1}{\sqrt{4 v^{2}-\left[\frac{c f_{t}}{\left(f_{c}+f_{\tau}\right)}\right]^{2}}}-\frac{r}{c}-\frac{d_{i}}{2 v},
$$

Based on (8) and (6), The PTRS phase is expressed as:

$$
\varphi_{i}\left(f_{\tau}, f_{t} ; r\right)=-4 \pi \frac{f_{\mathrm{c}}}{c} r \sqrt{\left(1+\frac{f_{\tau}}{f_{\mathrm{c}}}\right)^{2}-\frac{c^{2} f_{t}^{2}}{4 v^{2} f_{\mathrm{c}}{ }^{2}}}+\pi f_{t} \frac{d_{i}}{v}+2 \pi f_{t} \frac{r}{c}-\pi \frac{\left(f_{\mathrm{c}}+f_{\tau}\right)}{c} \frac{1}{2 r}\left(2 \frac{v}{c} r+d_{i}\right)^{2}
$$

The following work will be discussed based on (9). The first term is similar to that of monostatic SAS. The second term is introduced by the multi-receiver sampling. The third term is caused by stop-and-hop error. The last term includes the Doppler phase error and micro RCM, which are caused by the displaced distance between transmitter and receiver, together with the stop-and-hop error.

\section{Error Discussion}

\subsection{Signal Model Error}

To evaluate the approximation error, the accurate PSP and PTRS should be calculated. The numerical computing method is used to solve (5). Then, the accurate PTRS phase is given by:

$$
\Phi_{i}\left(f_{\tau}, f_{t} ; r\right)=-2 \pi\left(f_{\mathcal{C}}+f_{\tau}\right) \frac{R_{i}\left(t_{i \_n u} ; r\right)}{c}-2 \pi f_{t} t_{i \_n u},
$$

where $t_{i \_n u}$ is the numeric PSP.

The difference between (10) and (9) is the signal model error, which is shown as:

$$
\Delta \varphi_{i}\left(f_{\tau}, f_{t} ; r\right)=\Phi_{i}\left(f_{\tau}, f_{t} ; r\right)-\varphi_{i}\left(f_{\tau}, f_{t} ; r\right),
$$

Inspecting (11), the signal model error is a function of $f_{t}, f_{\tau}$, and $r$. Since the system shown in Figure 1 is characterized by cross-track invariance and along-track invariance, the targets located at synthetic aperture centers with different ranges are exploited to discuss the error variance.

When the SAS works with a narrow-bandwidth signal, the parameters are those in Table 1.

Based on (11) and the parameters shown in Table 1, we calculate the signal model error shown in Figure 2 for the first transmitter/receiver pair. By observing Figure 2a or other errors in Figure 2, the error magnitude increases with the Doppler frequency. On the one hand, the along-track variance of PCA error is not considered. Besides, the stop-and-hop error is approximately compensated with the range corresponding to the beam center, where the target is perpendicularly seen by the sonar and the Doppler frequency is zero. In other words, the along-track variance of the stop-and-hop error is not compensated either. From all errors in Figure 2, we find that the error magnitude slightly increases with range, 
as there is the residual stop-and-hop error [37]. In general, the error is slight when the receiver is close to the transmitter.

Table 1. SAS parameters with narrow bandwidth.

\begin{tabular}{ccc}
\hline Parameter & Value & Unit \\
\hline Platform velocity & 2 & $\mathrm{~m} / \mathrm{s}$ \\
Pulse repetition interval & 0.35 & $\mathrm{~s}$ \\
Signal bandwidth & 20 & $\mathrm{kHz}$ \\
Carrier frequency & 150 & $\mathrm{kHz}$ \\
Receiver array length & 1.4 & $\mathrm{~m}$ \\
Receiver width & 0.04 & $\mathrm{~m}$ \\
Transmitter width & 0.08 & $\mathrm{~m}$ \\
\hline
\end{tabular}

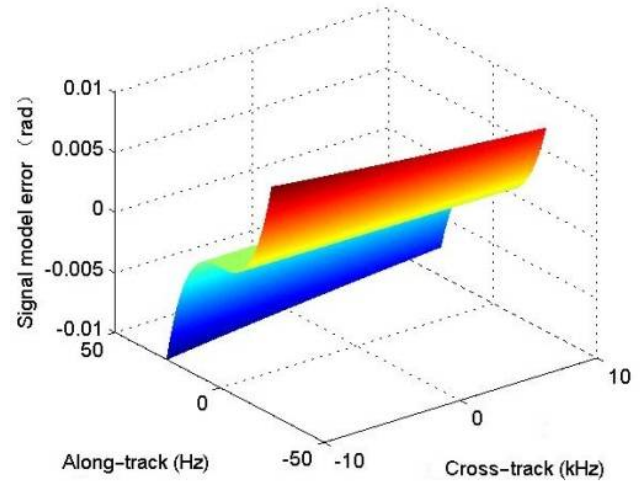

(a)

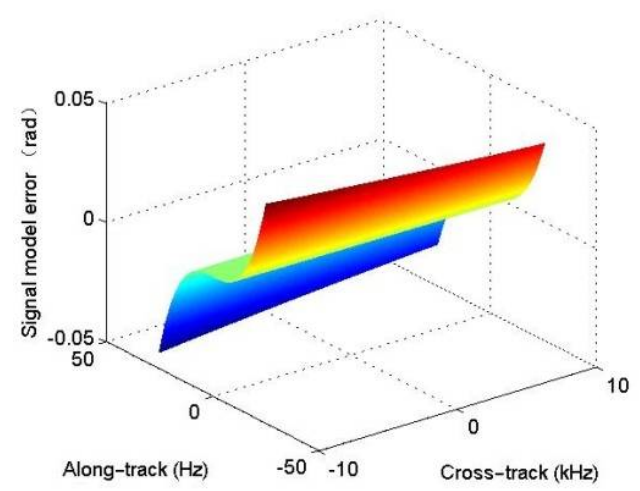

(c)

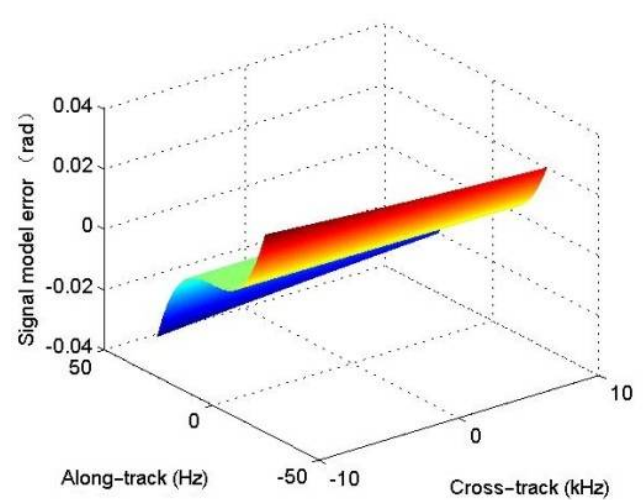

(b)

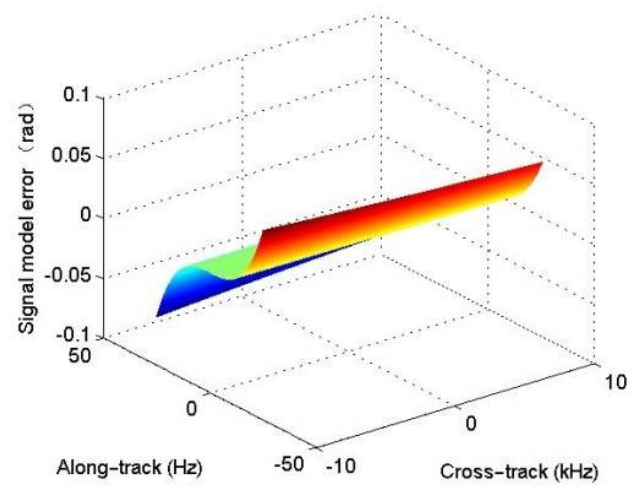

(d)

Figure 2. Signal model error for the first transmitter/receiver pair when the system works with narrow-bandwidth. (a) $r=40 \mathrm{~m}$; (b) $r=110 \mathrm{~m}$; (c) $r=180 \mathrm{~m}$; (d) $r=250 \mathrm{~m}$.

Considering the last transmitter/receiver pair, the signal model error is shown in Figure 3. Based on Figure 3, we can obtain the same conclusion drawn from Figure 2. Comparing Figure 2 to Figure 3, we find that the signal model error slightly depends on the displaced distance between transmitter and receiver. The maximum magnitude of the error is $0.1 \mathrm{rad}$, which is less than $\pi / 4[27,45]$. In other words, the signal model error can be neglected when the SAS system works with a narrow bandwidth. 


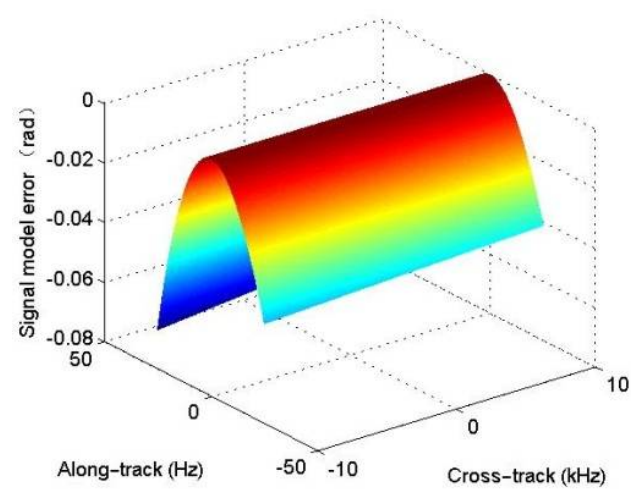

(a)

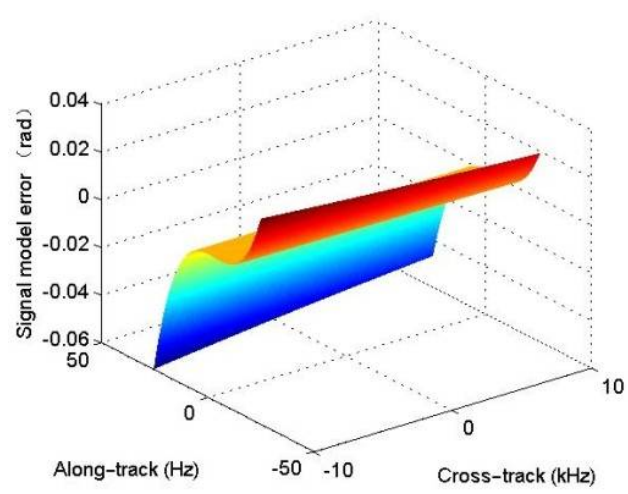

(c)

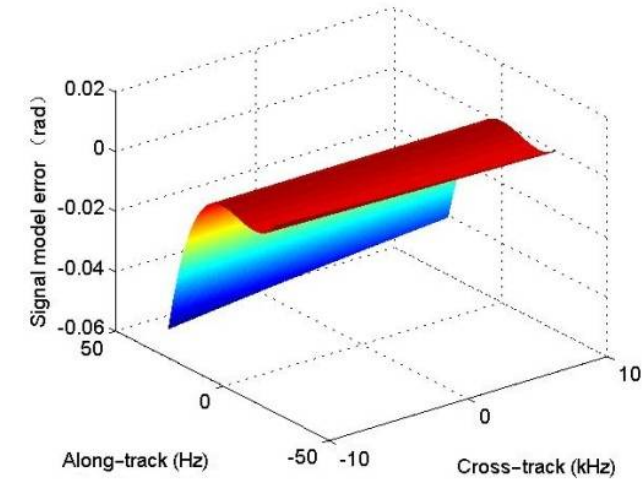

(b)

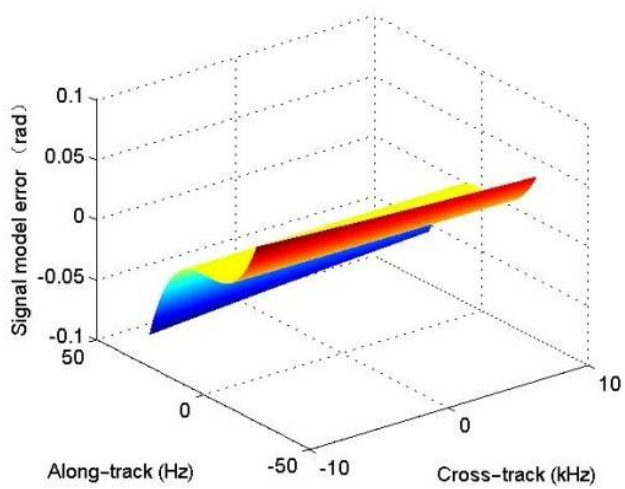

(d)

Figure 3. Signal model error for the last transmitter/receiver pair when the system works with narrow-bandwidth. (a) $r=40 \mathrm{~m}$; (b) $r=110 \mathrm{~m}$; (c) $r=180 \mathrm{~m}$; (d) $r=250 \mathrm{~m}$.

The SAS parameters are listed in Table 2 when the SAS works with a wide-bandwidth signal.

Table 2. SAS parameters with wide bandwidth.

\begin{tabular}{ccc}
\hline Parameter & Value & Unit \\
\hline Platform velocity & 2 & $\mathrm{~m} / \mathrm{s}$ \\
Pulse repetition interval & 0.35 & $\mathrm{~s}$ \\
Signal bandwidth & 60 & $\mathrm{kHz}$ \\
Carrier frequency & 150 & $\mathrm{kHz}$ \\
Receiver array length & 1.4 & $\mathrm{~m}$ \\
Receiver width & 0.04 & $\mathrm{~m}$ \\
Transmitter width & 0.08 & $\mathrm{~m}$ \\
\hline
\end{tabular}

The signal model errors for the first and last transmitter/receiver pairs are depicted in Figures 4 and 5, respectively. Comparing Figure 2 to Figure 4, we find that the signal bandwidth does not affect the signal model error. The same conclusion can be obtained based on Figures 3 and 5 .

In general, the signal model error is mostly independent of the signal bandwidth. Furthermore, the maximum magnitude of the signal model error is $0.1 \mathrm{rad}$. That is to say, the PTRS based on the PCA method can satisfy the high-resolution imagery. At this point, the PTRS would be deduced based on this PCA method. 


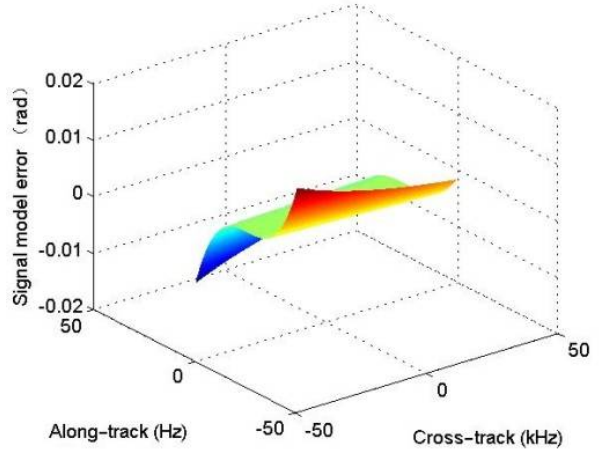

(a)

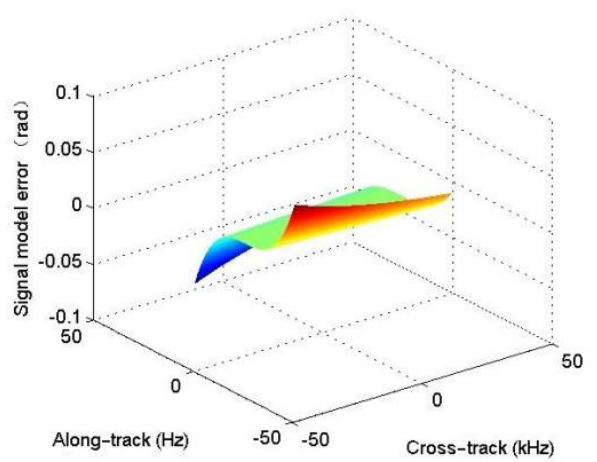

(c)

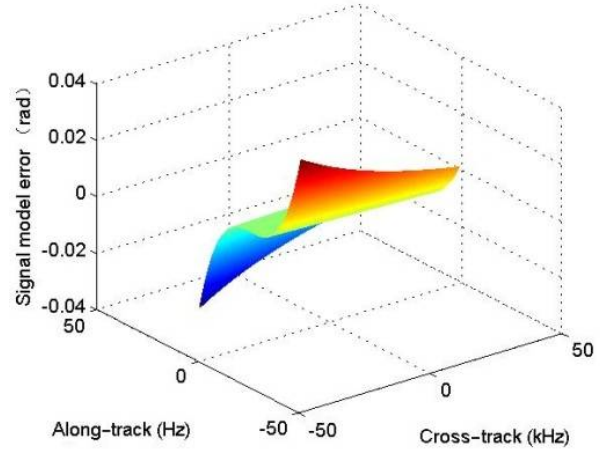

(b)

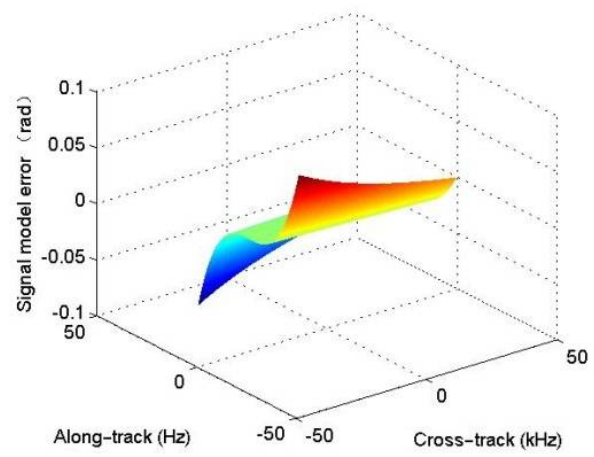

(d)

Figure 4. Signal model error for the first transmitter/receiver pair when the system works with wide-bandwidth. (a) $r=40 \mathrm{~m}$; (b) $r=110 \mathrm{~m}$; (c) $r=180 \mathrm{~m}$; (d) $r=250 \mathrm{~m}$.

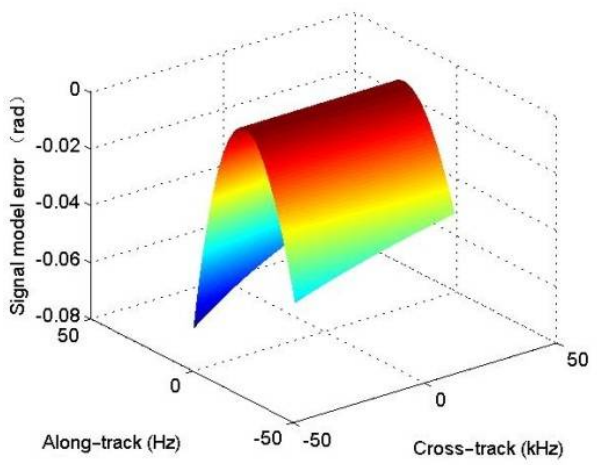

(a)

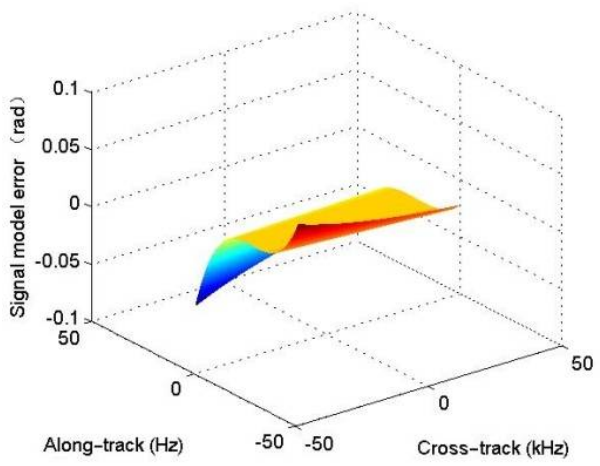

(c)

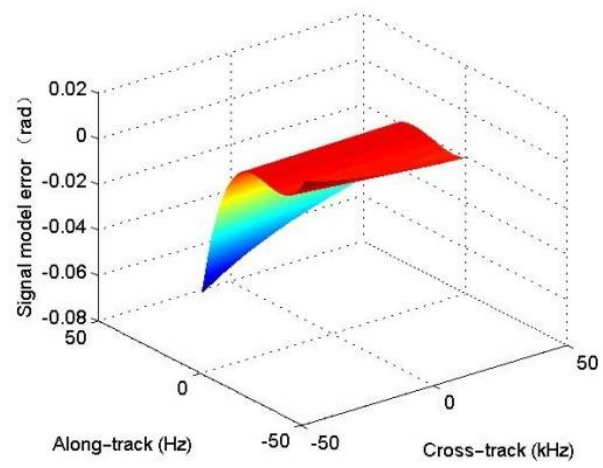

(b)

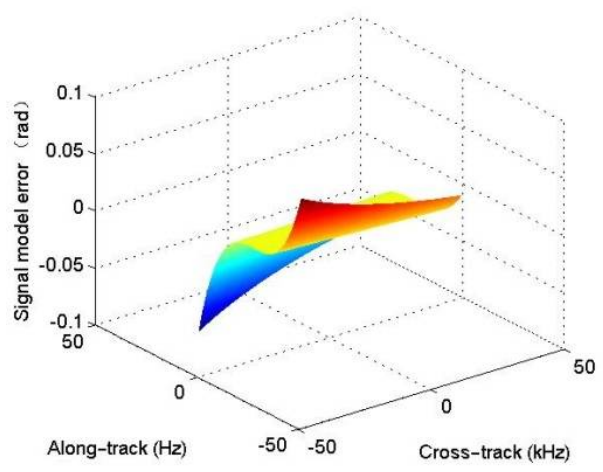

(d)

Figure 5. Signal model error for the last transmitter/receiver pair when the system works with wide-bandwidth. (a) $r=40 \mathrm{~m}$; (b) $r=110 \mathrm{~m}$; (c) $r=180 \mathrm{~m}$; (d) $r=250 \mathrm{~m}$. 


\subsection{PTRS Error}

The PTRS phase shown in (9) cannot be directly used by the imaging algorithm. It should be further formulated according to the R-D algorithm. Based on the R-D algorithm, the PTRS phase is expanded up to the second-order term with respect to instantaneous frequency $f_{\tau}$. Applying Taylor expansion to (9) yields:

$$
\hat{\varphi}_{i}\left(f_{\tau}, f_{t} ; r\right)=-\frac{4 \pi r \beta}{\lambda}-\frac{4 \pi r}{c \beta} f_{\tau}+2 \pi \frac{r \lambda}{c^{2}}\left(\frac{1}{\beta^{3}}-\frac{1}{\beta}\right) f_{\tau}^{2}+\pi f_{t} \frac{d_{i}}{v}+2 \pi f_{t} \frac{r}{c}-\pi \frac{\left(f_{\mathrm{c}}+f_{\tau}\right)}{c} \frac{1}{2 r}\left(2 \frac{v}{c} r+d_{i}\right)^{2},
$$

where $\beta=\sqrt{1-\frac{c^{2} f_{t}^{2}}{4 v^{2} f_{c}^{2}}}$. The first term in (12) denotes the along-track modulation. The second term is RCM. The third term represents the quadratic coupling between crosstrack and along-track dimensions. It is equivalent to a chirp modulation in the cross-track dimension. The remaining terms are the same as the ones in (9).

The approximation error between (9) and (13) is given by:

$$
\Delta \hat{\varphi}_{i}\left(f_{\tau}, f_{t} ; r\right)=\varphi_{i}\left(f_{\tau}, f_{t} ; r\right)-\hat{\varphi}_{i}\left(f_{\tau}, f_{t} ; r\right),
$$

We firstly discuss the PTRS error under the case of the narrow-bandwidth signal. Based on (13) and Table 1, the PTRS errors for the first and last transmitter/receiver pairs are shown in Figures 6 and 7, respectively.

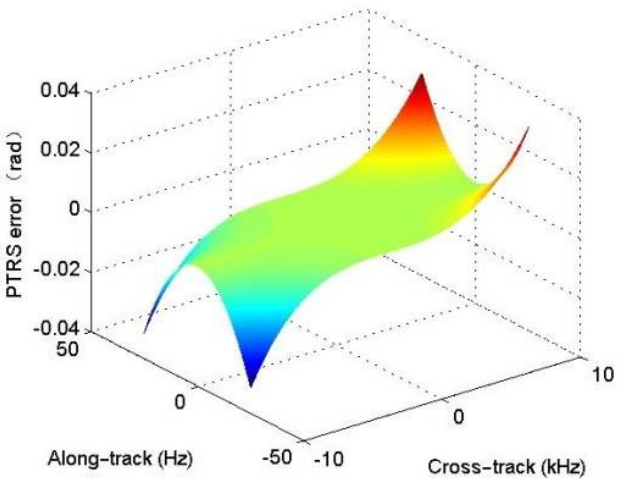

(a)

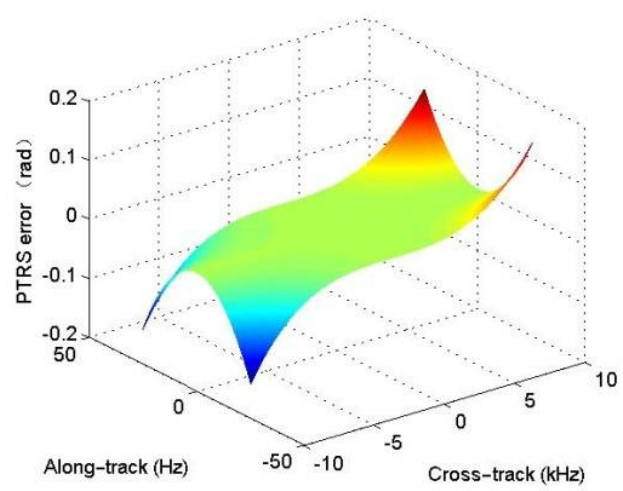

(c)

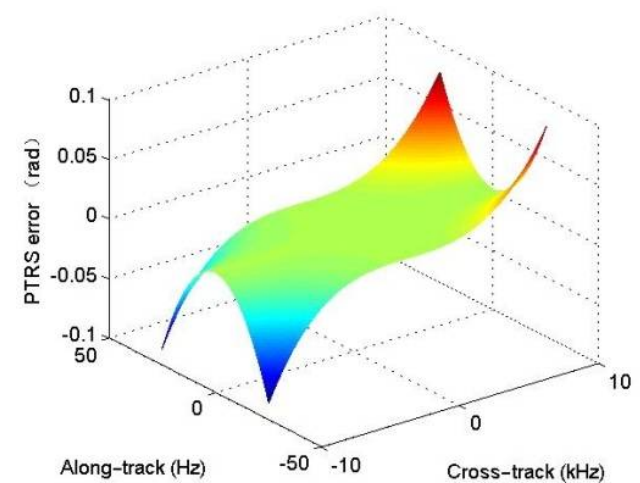

(b)

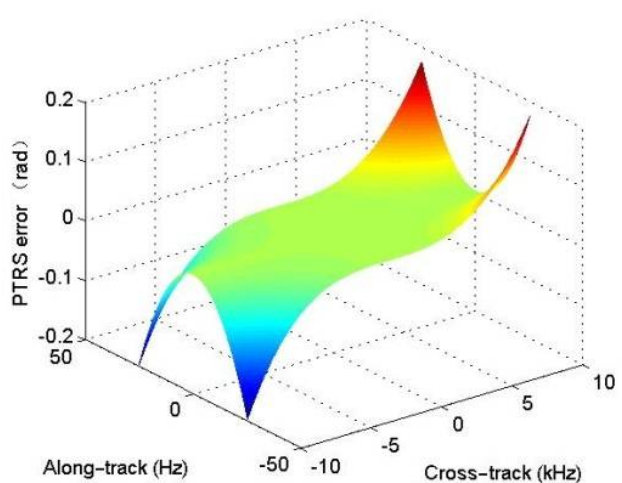

(d)

Figure 6. PTRS error for the first transmitter/receiver pair when the system works with a narrowbandwidth. (a) $r=40 \mathrm{~m}$; (b) $r=110 \mathrm{~m}$; (c) $r=180 \mathrm{~m}$; (d) $r=250 \mathrm{~m}$. 


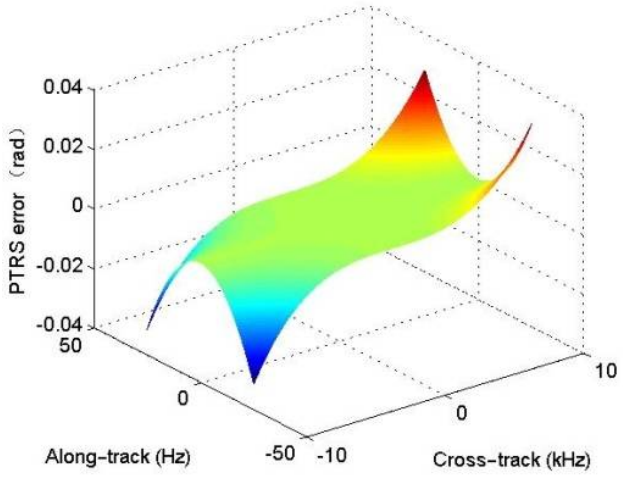

(a)

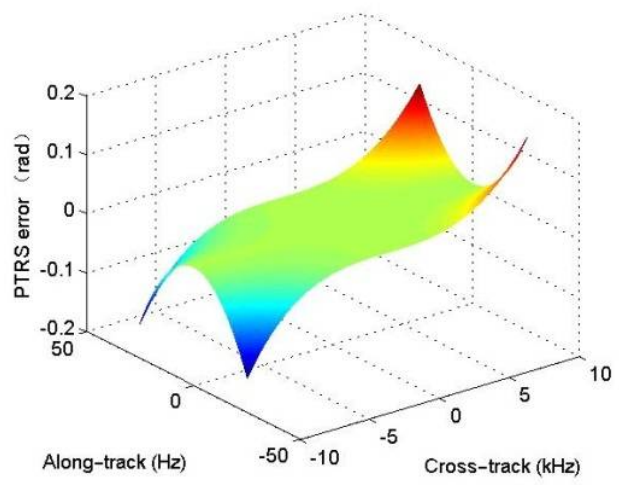

(c)

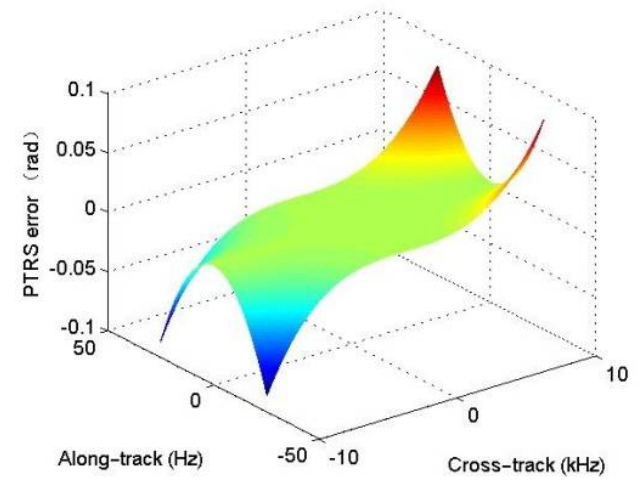

(b)

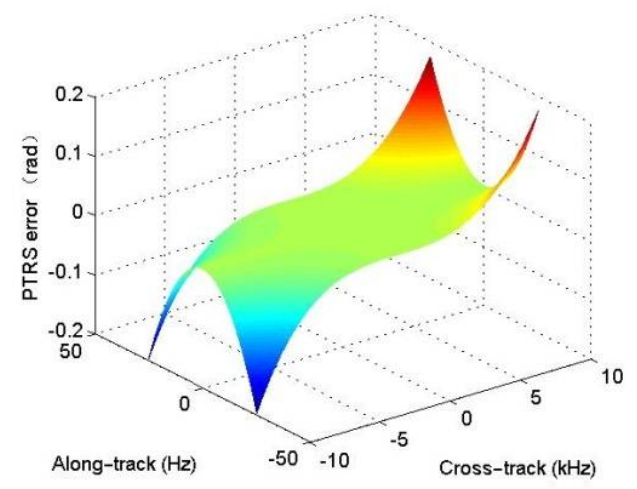

(d)

Figure 7. PTRS error for the last transmitter/receiver pair when the system works with a narrowbandwidth. (a) $r=40 \mathrm{~m}$; (b) $r=110 \mathrm{~m}$; (c) $r=180 \mathrm{~m}$; (d) $r=250 \mathrm{~m}$.

From Figures 6 and 7, we find that the magnitude of the PTRS error increases with instantaneous frequency, as the Taylor expansion is conducted around the zero-frequency. Moreover, the error slightly increases with range. Based on Figures 6 and 7, the PTRS error can be negligible when the SAS works with the case of a narrow-bandwidth signal. Furthermore, the displaced distance between the transmitter and receiver does not affect the PTRS error.

We now discuss the PTRS error when the SAS uses the wide-bandwidth signal. Based on parameters in Table 2, the PTRS errors under the case of the wide bandwidth are depicted in Figures 8 and 9. When the signal bandwidth is $60 \mathrm{kHz}$, the maximum magnitude of PTRS error is $6 \mathrm{rad}$, which would seriously affect SAS performance.

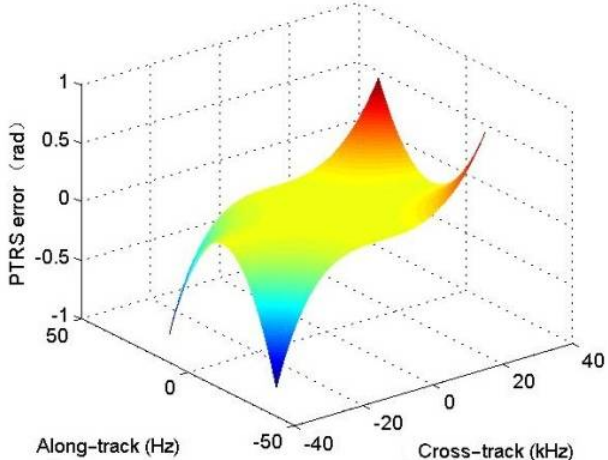

(a)

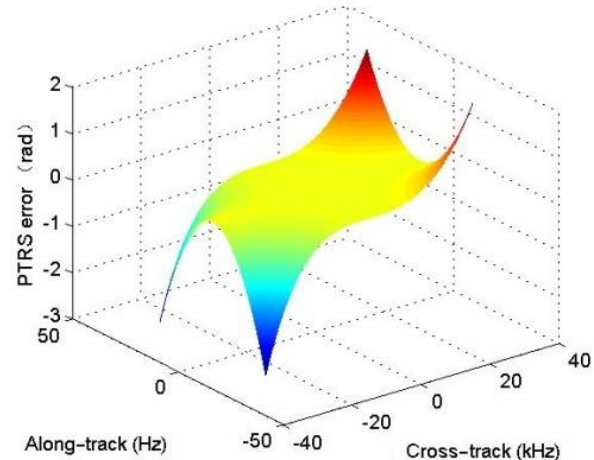

(b)

Figure 8. Cont. 


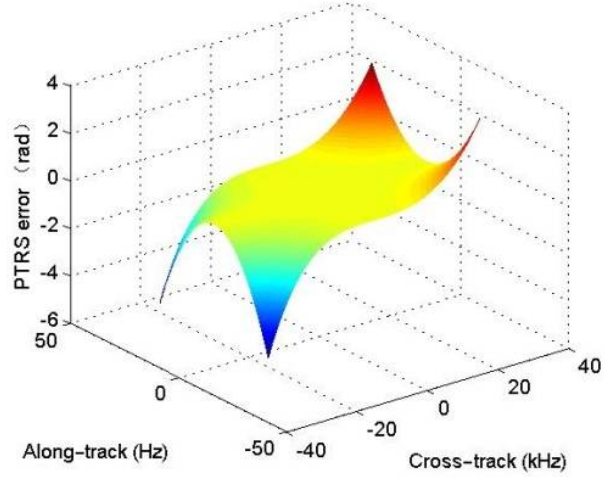

(c)

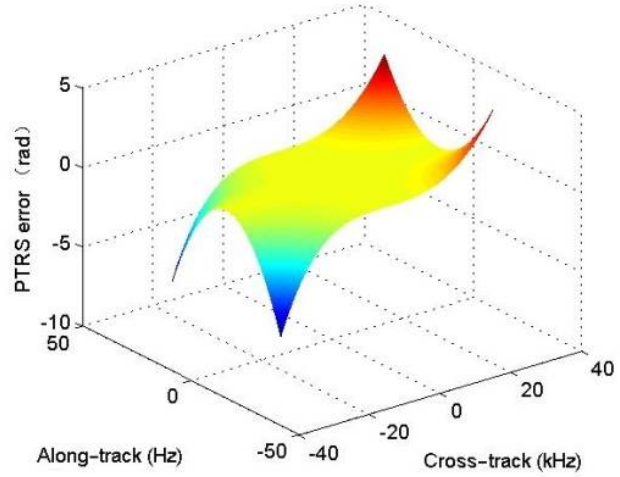

(d)

Figure 8. PTRS error for the first transmitter/receiver pair when the system works with a widebandwidth. (a) $r=40 \mathrm{~m}$; (b) $r=110 \mathrm{~m}$; (c) $r=180 \mathrm{~m}$; (d) $r=250 \mathrm{~m}$.

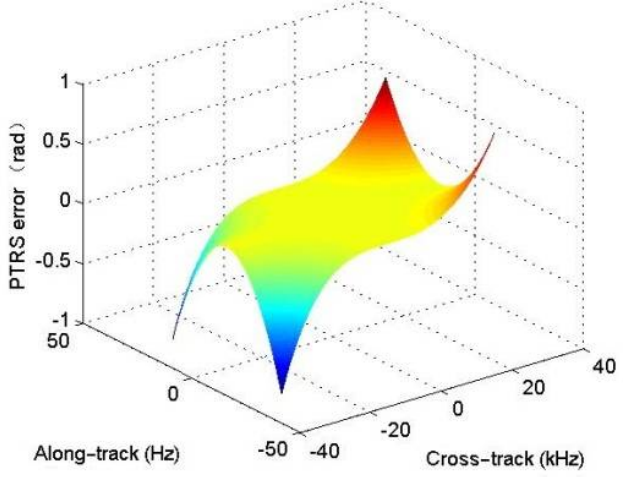

(a)

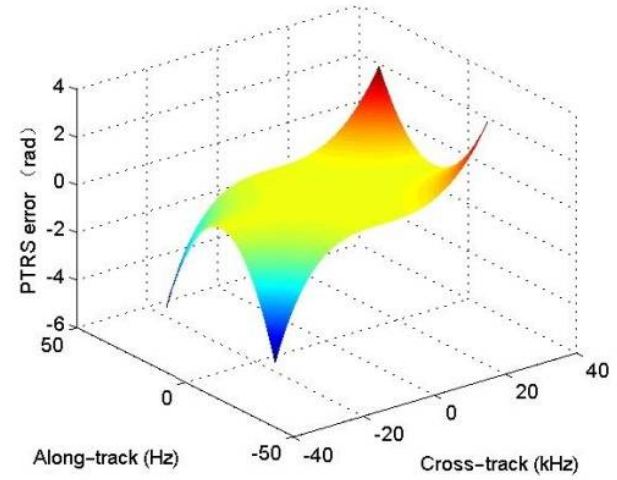

(c)

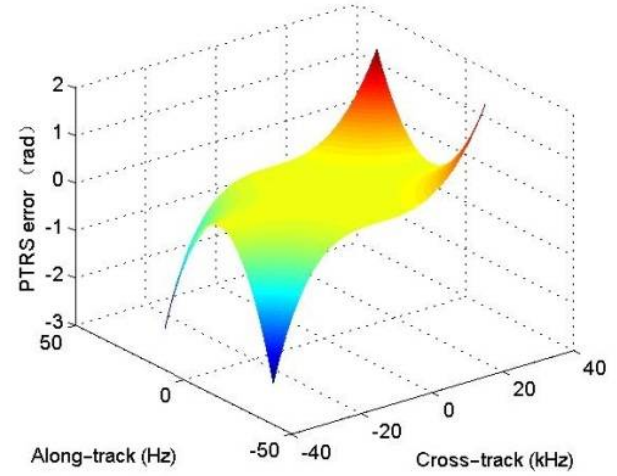

(b)

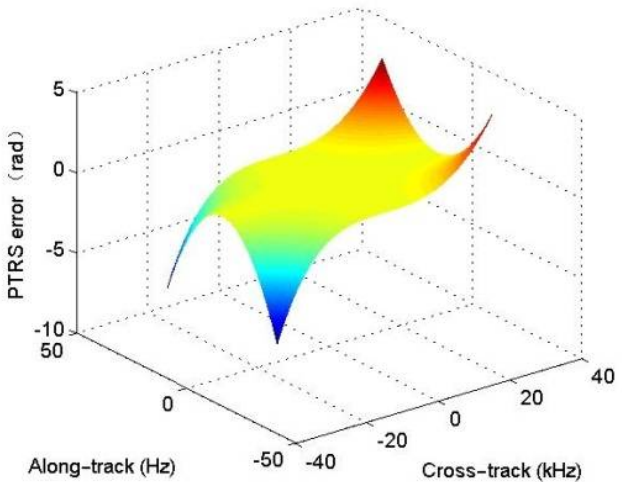

(d)

Figure 9. PTRS error for the last transmitter/receiver pair when the system works with a widebandwidth. (a) $r=40 \mathrm{~m}$; (b) $r=110 \mathrm{~m}$; (c) $r=180 \mathrm{~m}$; (d) $r=250 \mathrm{~m}$.

Generally speaking, the PTRS error significantly depends on the signal bandwidth. When the bandwidth is narrow, the PTRS error can be neglected. However, it cannot be neglected when the SAS system works with a wide-bandwidth signal. The reason is that the Taylor approximation just keeps the second-order term.

\subsection{Residual Quadratic Coupling Error}

With the R-D algorithm, secondary range compression (SRC) is conducted to deal with the quadratic coupling between cross-track and along-track dimensions. Inspecting the quadratic coupling in (12), it is a function of range, Doppler frequency, and instantaneous frequency. To simplify the compensation, quadratic coupling at the reference range is 
often exploited by the traditional R-D algorithm [27]. This operation would further lead to approximation error. This section discusses this error, which is shown as:

$$
\varphi_{\mathrm{src}}\left(f_{\tau}, f_{t} ; r\right)=2 \pi \frac{r \lambda}{c^{2}}\left(\frac{1}{\beta^{3}}-\frac{1}{\beta}\right) f_{\tau}^{2}-2 \pi \frac{r_{\text {ref }} \lambda}{c^{2}}\left(\frac{1}{\beta^{3}}-\frac{1}{\beta}\right) f_{\tau}^{2},
$$

where $r_{\text {ref }}$ denotes the reference range.

Based on (14) and Table 1, the residual quadratic coupling error is shown in Figure 10 when the system works with the narrow-bandwidth signal. Here, the reference range is the scene center. When the targets are far away from the reference range, the error magnitude is noticeable. Figure 10a enhances this conclusion. When the targets are close to the reference range, the error magnitude is small. In general, the error magnitude decreases with the difference between the target range and the reference range.

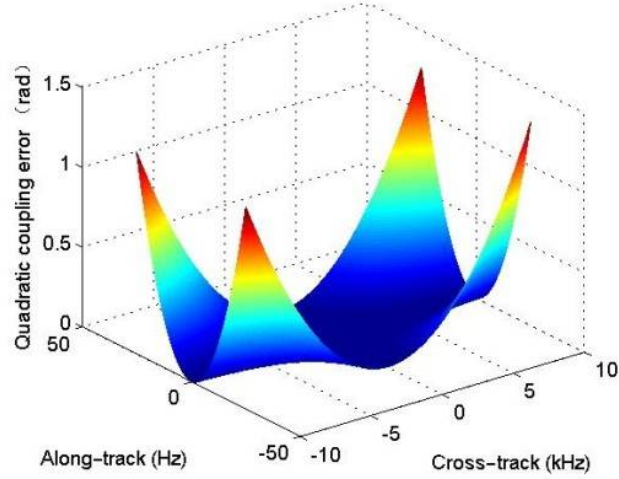

(a)

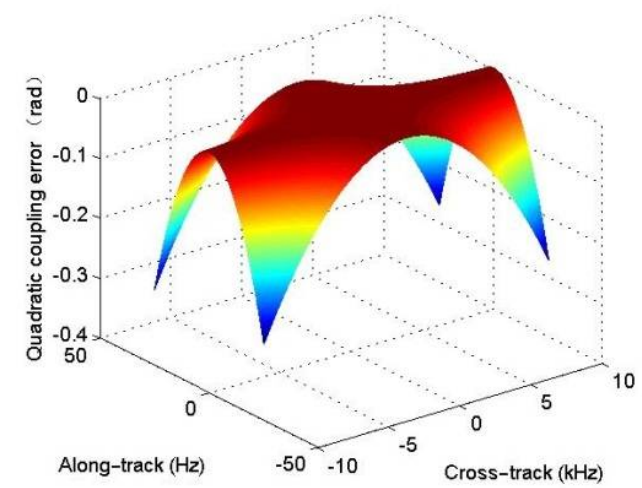

(c)

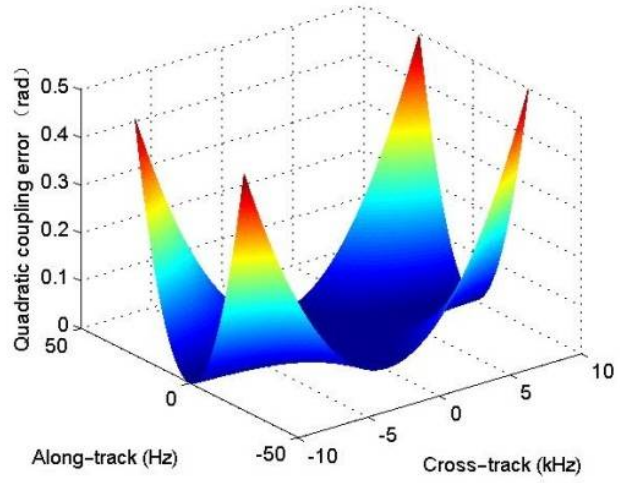

(b)

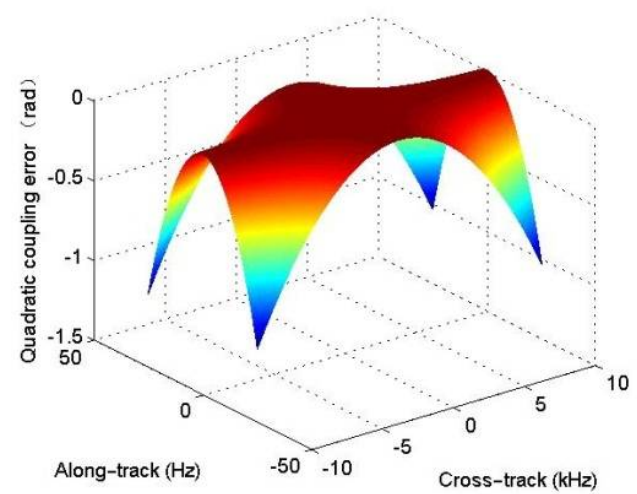

(d)

Figure 10. Residual quadratic coupling error when the system works with a narrow-bandwidth signal. (a) $r=40 \mathrm{~m}$; (b) $r=110 \mathrm{~m}$; (c) $r=180 \mathrm{~m}$; (d) $r=250 \mathrm{~m}$.

We now focus on the error with the wide-bandwidth signal. Based on the parameters in Table 2, the error is shown in Figure 11. We can obtain the same conclusions drawn from Figure 12. Comparing Figure 10 to Figure 11, we find that the residual quadratic coupling error highly depends on the signal bandwidth.

Summarily, the residual quadratic coupling error increases with the signal bandwidth. With the narrow-bandwidth case, this error is small. However, the wide-bandwidth can lead to a large residual error. 


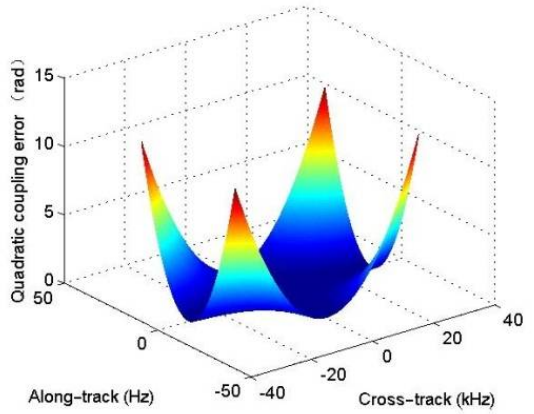

(a)

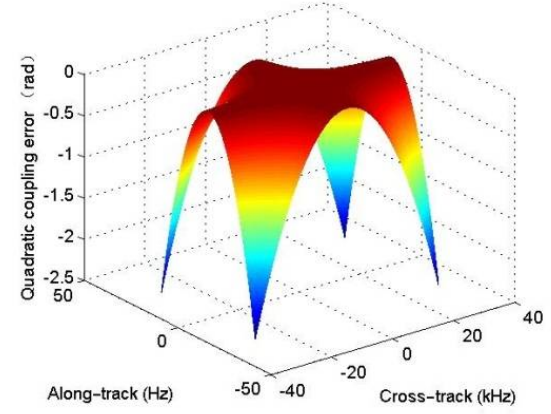

(c)

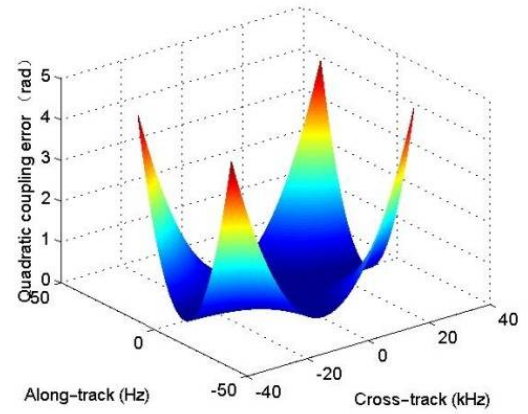

(b)

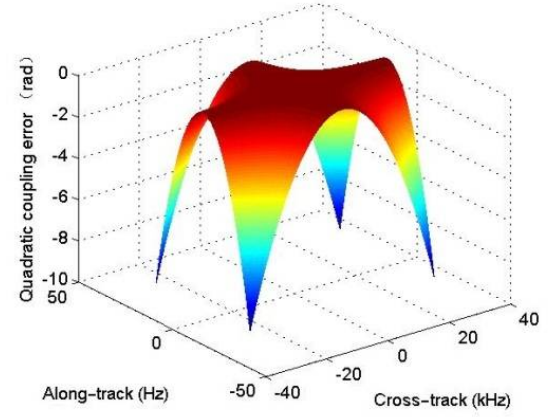

(d)

Figure 11. Residual quadratic coupling error when the system works with a wide-bandwidth. (a) $r=40 \mathrm{~m}$; (b) $r=110 \mathrm{~m}$; (c) $r=180 \mathrm{~m}$; (d) $r=250 \mathrm{~m}$.

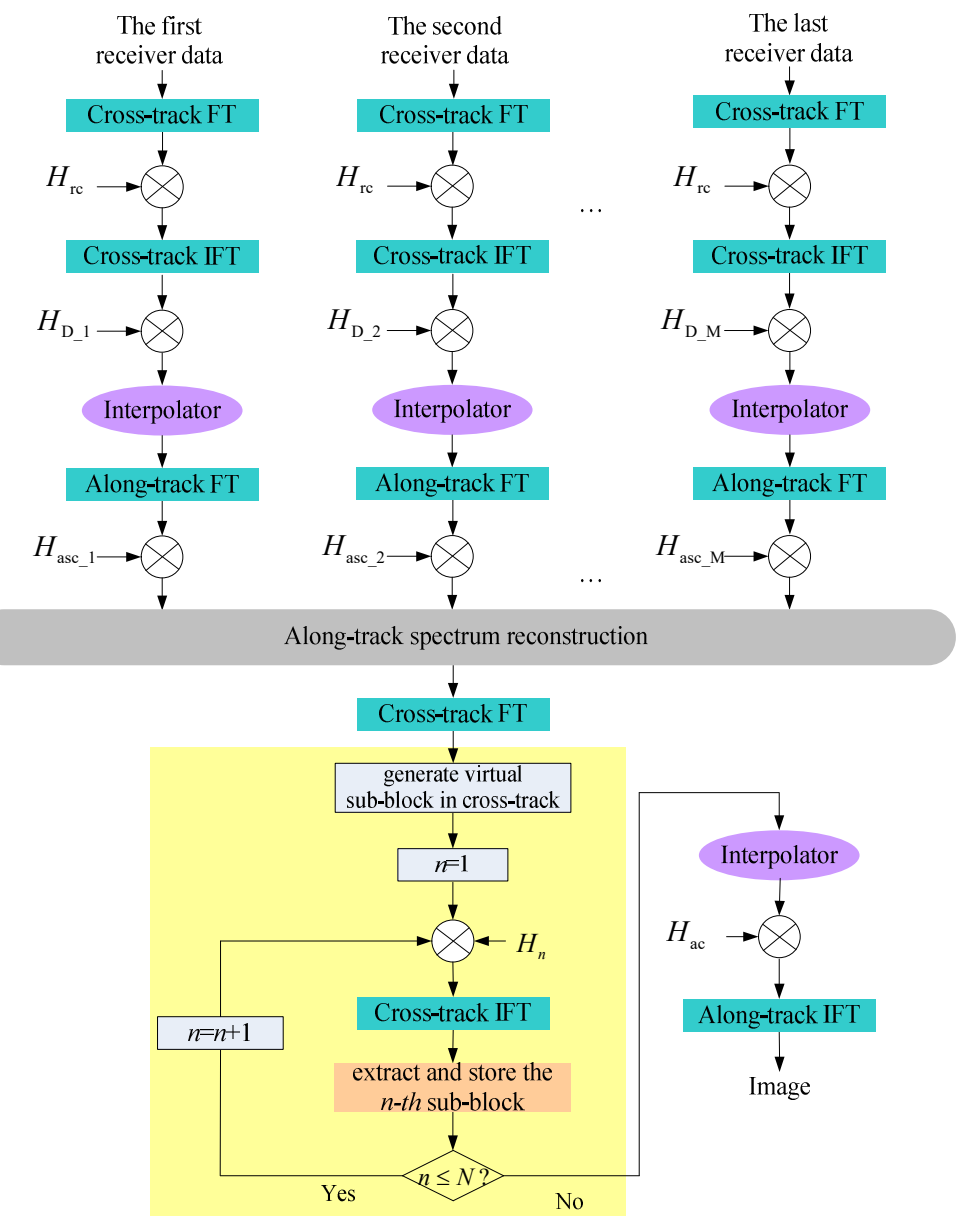

Figure 12. Block diagram of improved R-D algorithm. 


\section{Imaging Algorithm}

\subsection{Imagery}

To compensate for the PTRS error and residual quadratic coupling error, this section discusses an improved multi-receiver SAS R-D algorithm based on (9) and (12).

For each receiver data, the echo signal should be transformed into the frequency domain in the cross-track dimension. The cross-track compression is carried out based on the filtering function expressed as:

$$
H_{r c}=\operatorname{conj}\left\{P\left(f_{\tau}\right)\right\},
$$

where $\operatorname{conj}(\cdot)$ denotes the conjugated operation.

After cross-track compression, the data is transformed into the time domain. The Doppler phase error is compensated by:

$$
H_{D_{-} i}=\exp \left\{j \pi f_{\mathrm{c}} \frac{1}{2 c r}\left(2 \frac{v}{c} r+d_{i}\right)^{2}\right\},
$$

When the Doppler phase error is compensated, the sinc-interpolator is exploited to correct the micro RCM, which is shown as:

$$
\Delta_{i}=\frac{1}{4 c r}\left(2 \frac{v}{c} r+d_{i}\right)^{2},
$$

Then, the data is transformed into the Doppler frequency domain in the along-track dimension. The second term introduced by the multi-receiver sampling is compensated by the filtering function given by:

$$
H_{\text {asc } \_ \text {i }}=\exp \left\{j \pi f_{t} \frac{d_{i}}{v}\right\},
$$

After processing each receiver data, the spectrum corresponding to each receiver data is coherently superposed in the Doppler frequency domain. With this operation, the signal, which is equivalent to traditional monostatic SAS, is obtained. The signal is transformed into a 2-D frequency domain based on cross-track FT. The data is divided into $N$ sub-blocks in the cross-track dimension. For the $n$-th $(n \in[1, N])$ sub-block, the higher-order phase error is compensated by:

$$
H_{\mathrm{n}}=\exp \left\{j 4 \pi \frac{f_{\mathrm{c}}}{c} r \sqrt{\left(1+\frac{f_{\tau}}{f_{\mathrm{c}}}\right)^{2}-\frac{c^{2} f_{t}{ }^{2}}{4 v^{2} f_{\mathrm{c}}{ }^{2}}}-j \frac{4 \pi r \beta}{\lambda}+j \frac{4 \pi r}{c \beta} f_{\tau}\right\},
$$

With this operation, the SRC considering the cross-track variance of the second-order term is further conducted. The data is transformed into the time domain in cross-track. We extract and store this sub-block. The error compensation of the remaining sub-blocks should be processed based on (19). When the higher-order errors of all sub-blocks are compensated, all sub-blocks are coerced into a new signal.

The sinc-interpolator is exploited to perform RCMC, and the RCM is shown as:

$$
\Delta_{\mathrm{RCM}}=\frac{2 r}{c \beta},
$$

After RCMC, the along-track compression is conducted based on the filtering function given by:

$$
H_{\mathrm{ac}}=\exp \left\{j \frac{4 \pi r \beta}{\lambda}-j 2 \pi f_{t} \frac{r}{c}\right\},
$$

With this operation, the along-track shifting introduced by stop-and-hop error is simultaneously compensated. 
Based on the method described in this section, the block diagram of our method is depicted in Figure 12. Compared to the traditional R-D algorithm, the presented method has an additional step to compensate for the residual phase error introduced by the secondorder Taylor expansion. The compensation of phase error is depicted by the pale-yellow area in Figure 12.

\subsection{Computation Load}

In this section, we mainly discuss the computation load of the presented method. For clarity, the computation load of basic operations is listed in Table 3. In general, FT, IFT, multiplication, and sinc-based interpolation are used by the R-D algorithm. For clarity, we suppose that there are $N_{r}$ sampling points in the cross-track dimension. $N_{a}$ sampling points are collected in the along-track dimension. $\rho$ represents the length of the interpolation kernel function, which is a sinc-function in this paper.

Table 3. Computation load of basic operations.

\begin{tabular}{ccc}
\hline Operations & Data Size & Computation Load \\
\hline Cross-track FT/IFT & $N_{r}$ & $\mathrm{O}\left(1.5 N_{r} \log _{2} N_{r}\right)$ \\
Cross-track FT/IFT & $N_{a} \times N_{r}$ & $\mathrm{O}\left(1.5 N_{a} \cdot N_{r} \log _{2} N_{r}\right)$ \\
Along-track FT/IFT & $N_{a} \times N_{r}$ & $\mathrm{O}\left(1.5 N_{a} \cdot N_{r} \log _{2} N_{a}\right)$ \\
Multiplication & $N_{a} \times N_{r}$ & $\mathrm{O}\left(N_{a} \cdot N_{r}\right)$ \\
Sinc-based interpolation & $\rho$ & $\mathrm{O}(2 \rho-1)$ \\
\hline
\end{tabular}

Table 4 lists the basic operations used by the traditional R-D algorithm, presented method, and BP algorithm. We find that the BP algorithm is very time-consuming due to interpolation. However, it can provide high-resolution images, which can be used as the criteria to evaluate the performance of fast imaging algorithms. Compared to the traditional method, the efficiency of the presented method is lowered, as more time is needed to compensate for the approximation error based on the sub-block processing method in the cross-track dimension. Consequently, our method can provide high-resolution images when the wideband signal is used by the SAS system.

Table 4. Basic operations used by three imaging algorithms.

\begin{tabular}{cccc}
\hline Operations & Presented Method & Traditional Method & BP Algorithm \\
\hline Cross-track FT/IFT & $2 M+1+N$ & $2 M+2$ & 2 \\
Along-track FT/IFT & $M+1$ & $M+1$ & 0 \\
Multiplication & $3 M+N+1$ & $3 M+1$ & $N_{a} \times N_{r}$ \\
Interpolation & $M+1$ & $M+1$ & $N_{a}^{2} \times N_{r}$ \\
\hline
\end{tabular}

\section{Experiment Results}

\subsection{Simulations}

We carry out simulations to validate the presented method in this section. We suppose that there are three ideal targets marked by T1, T2, and T3, which are shown in Figure 13. The vertical axis is the moving direction of the sonar platform, and it is also called the alongtrack dimension or azimuth dimension. The horizontal axis is also called the cross-track dimension or range dimension. When we perform simulations, no weighting functions are used in the cross-track and along-track dimensions. That is to say, rectangle functions are used when the echoed signal is simulated. With our simulations, two cases, including the narrow-bandwidth signal and wide-bandwidth signal, are considered separately. To fairly compare the imaging performance, the only difference of SAS parameters between narrow and wide bandwidth cases lies in the signal bandwidth. That is to say, the other SAS parameters between both cases are identical. With this operation, the imaging results can clearly indicate which parameter affects the SAS imaging performance. The reference range used by secondary range compression is the center range across the whole imaging swath. 


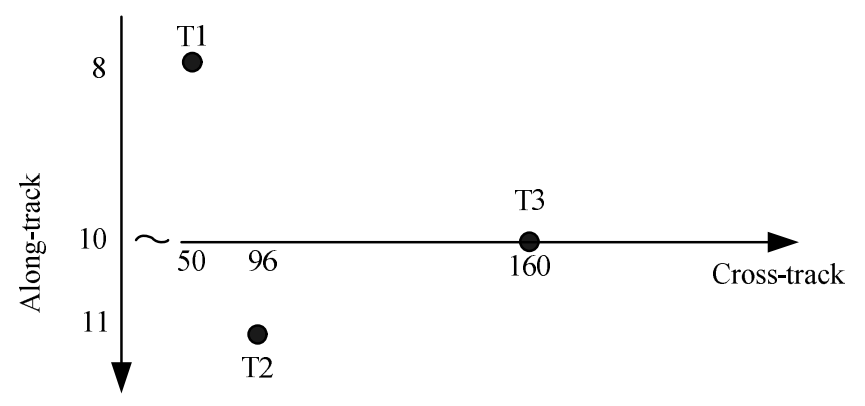

Figure 13. Distribution of simulation scene.

\subsubsection{Results with Narrow-Bandwidth Case}

Firstly, the simulations based on the narrow-bandwidth are focused, and the SAS parameters are listed in Table 1 . In practice, the imaging algorithm is the filtering of the echoed signal. The BP algorithm is based on the accurate system transfer function. The basic processing of fast imaging algorithms is to design filtering functions, which are infinitely close to the accurate system transfer function used by the BP algorithm [42-44]. At this point, the imaging results based on the BP algorithm [42-44] are often used as the criteria. Here, the traditional R-D algorithm [38], BP algorithm [42-44], and presented method are exploited to deal with the simulated data. After synthetic aperture image formation, the imaging results are shown in Figure 14. It can be clearly found that the traditional method and presented method can obtain the same results as the BP algorithm.
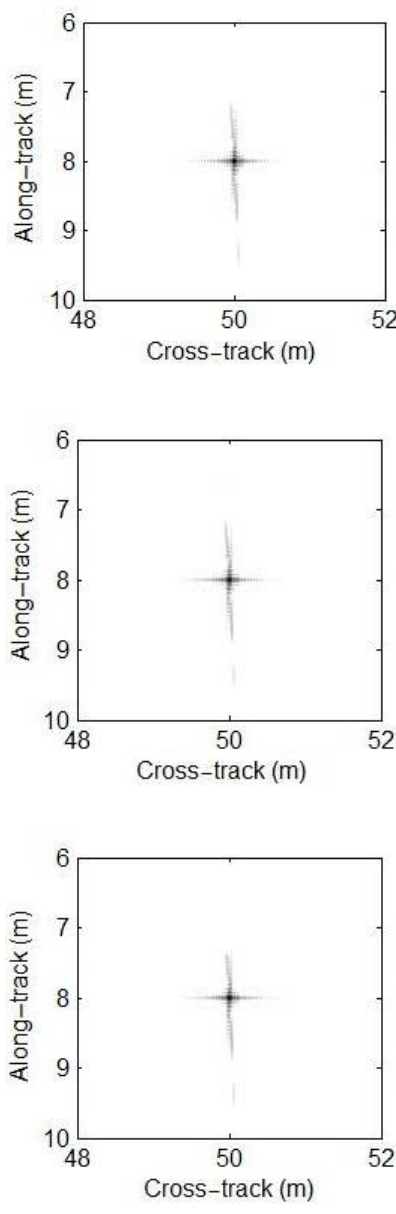

Figure 14. Imaging results with the case of a narrow-bandwidth signal. (a) Traditional method; (b) presented method; (c) BP algorithm.

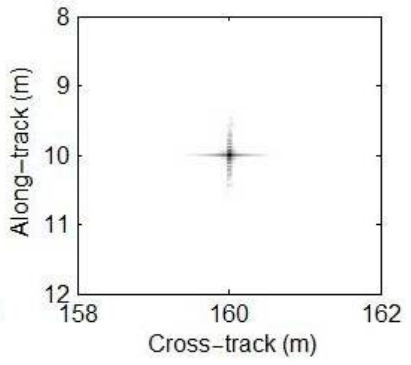

(a)

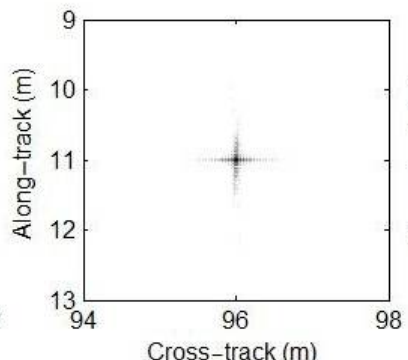

(b)

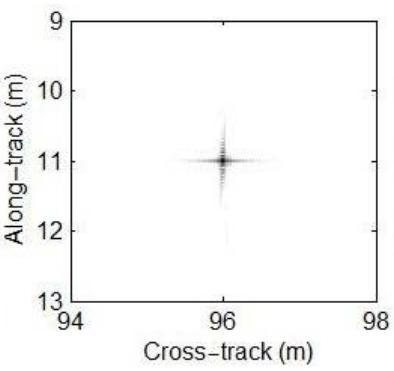

(c)
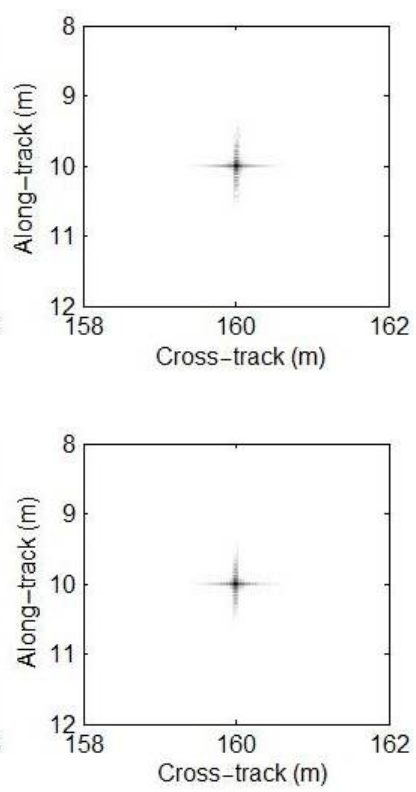
The along-track slices depicted in Figure 15 are further compared. In general, three methods can obtain the same results when the SAS system works with a narrow-bandwidth signal. This is consistent with the conclusions drawn from Section 3, as the PTRS error and residual quadratic coupling error do not noticeably affect the SAS imaging performance.

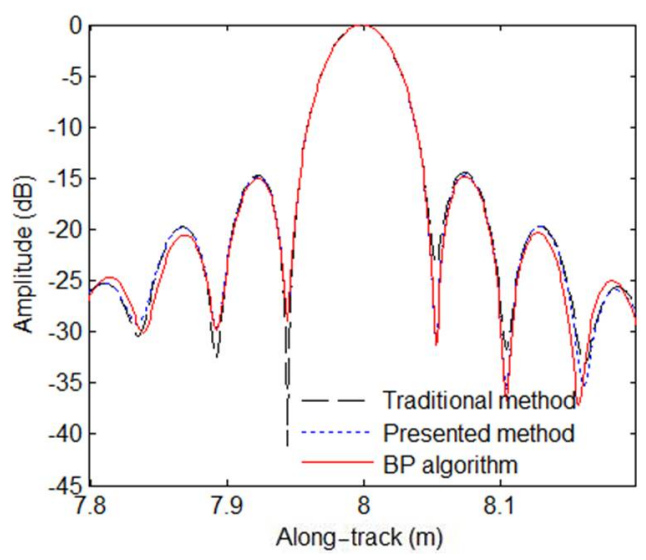

(a)

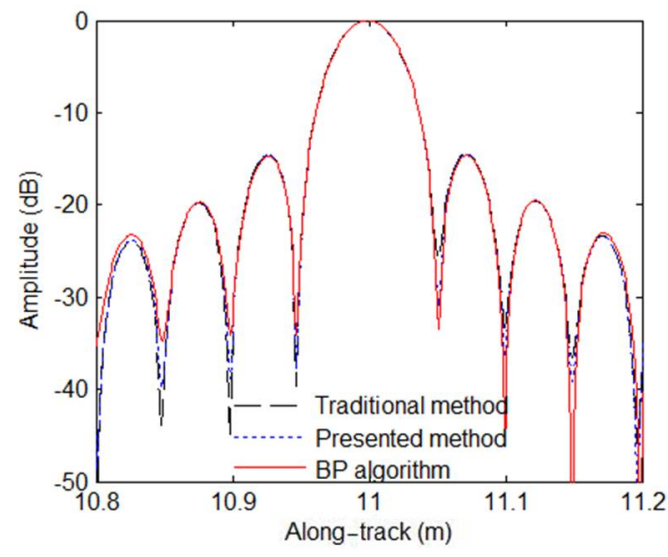

(b)

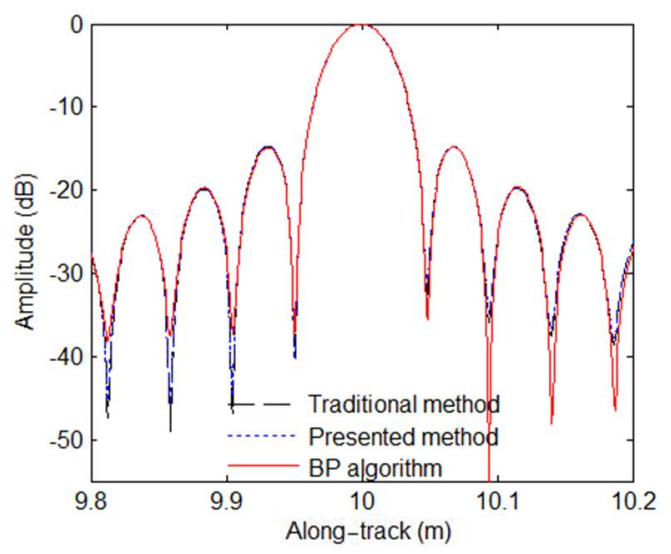

(c)

Figure 15. Along-track slices with the case of a narrow-bandwidth signal. (a) T1; (b) T2; (c) T3.

The peak sidelobe ratio (PSLR) and integrated sidelobe ratio (ISLR) listed in Table 5 are further compared. Based on Table 5, it can clearly be seen that the performance loss of the traditional R-D algorithm is slight. Summarily, both the presented method and traditional R-D algorithm can well reconstruct three targets. At this point, we conclude that the traditional R-D algorithm [38] can be directly applied to a narrow-bandwidth SAS system without loss of imaging performance.

Table 5. Comparison of imaging quality with a narrow-bandwidth case.

\begin{tabular}{cccc}
\hline Method & PLSR (dB) & ISLR (dB) & Target \\
\hline Traditional method & -14.47 & -8.04 & \\
Presented method & -14.66 & -8.17 & $\mathrm{~T} 1$ \\
BP algorithm & -14.81 & -8.27 & \\
\hline Traditional method & -14.47 & -8.79 & $\mathrm{~T} 2$ \\
Presented method & -14.52 & -8.88 & \\
BP algorithm & -14.64 & -8.90 & $\mathrm{~T} 3$ \\
\hline Traditional method & -14.70 & -9.76 & \\
Presented method & -14.75 & -9.81 & \\
BP algorithm & -14.87 & -9.88 & \\
\hline
\end{tabular}




\subsubsection{Results with Wide-Bandwidth Case}

We now concentrate on when the SAS system uses a wide-bandwidth signal. The SAS parameters can be found in Table 2. Based on the traditional R-D algorithm [38], presented method, and BP algorithm [42-44], the processing results are depicted in Figure 16. Inspecting Figure 16a, the performance of the traditional method is seriously affected by the phase error, which is mainly caused by PTRS error and residual quadratic coupling error. Comparing Figure $16 \mathrm{~b}$ to Figure $16 \mathrm{a}$, the proposed method can significantly improve the imaging performance. Based on Figure 16c, the performance of the presented method agrees with that of the BP algorithm. This indicates that the presented method can still reconstruct targets when the wide-bandwidth signal is exploited by the SAS system.
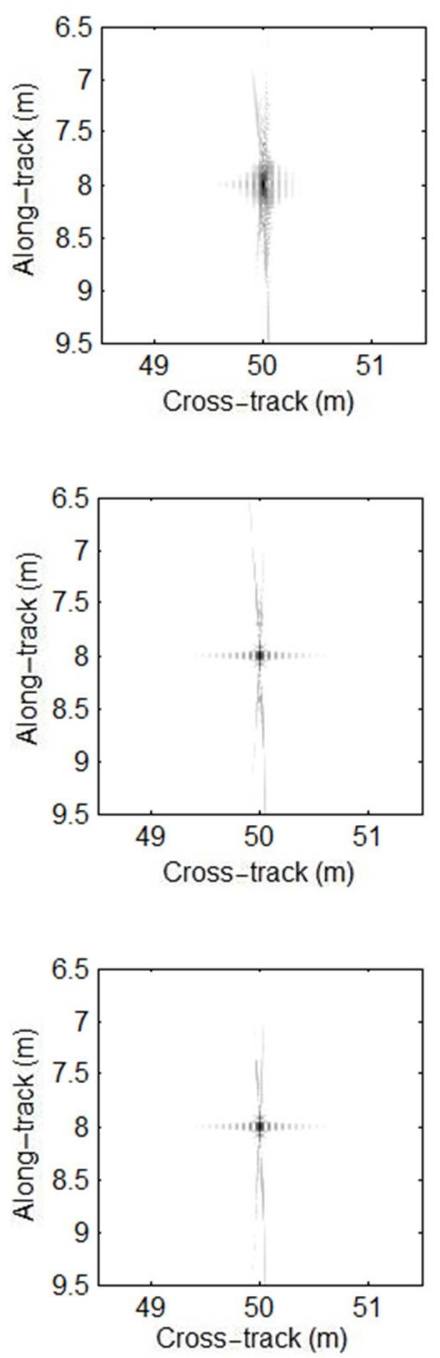

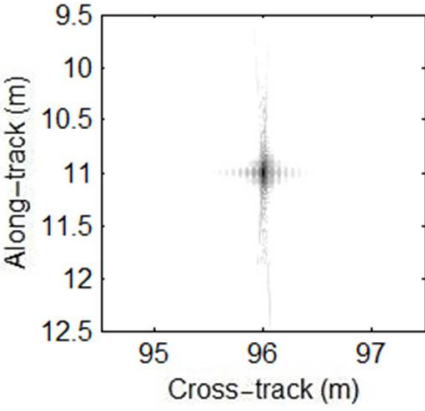

(a)

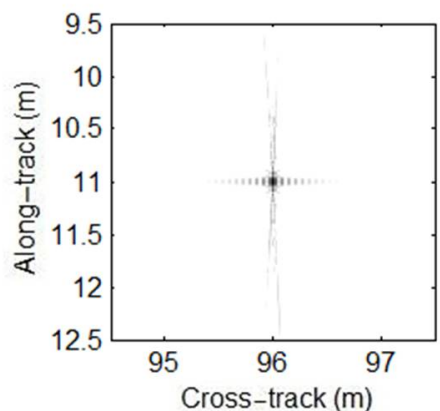

(b)

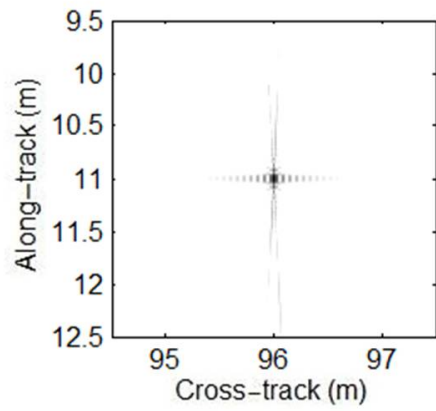

(c)
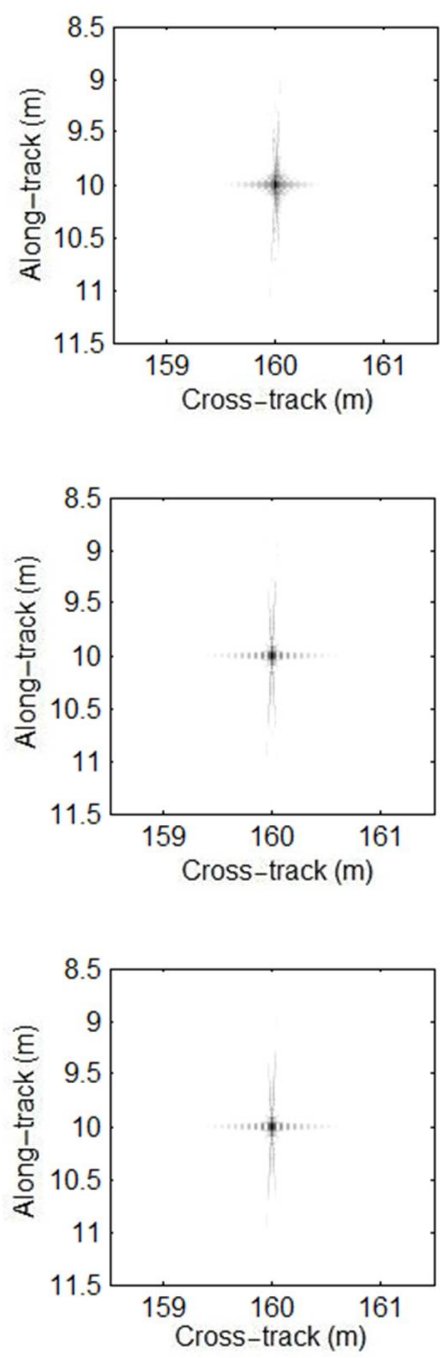

Figure 16. Imaging results with the case of a wide-bandwidth signal. (a) Traditional method; (b) presented method; (c) BP algorithm.

To visually compare the imaging performance, the along-track slices are shown in Figure 17. Based on Figure 17a,b, we clearly find that the performance of the traditional method is defocused. Fortunately, the slices of the presented method are mostly the same as for slices of the BP algorithm [42-44]. This enhances conclusions drawn from Figure 16. 


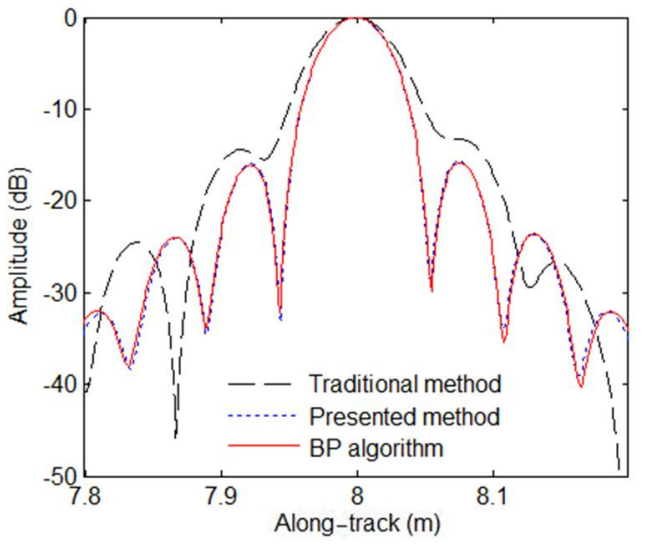

(a)

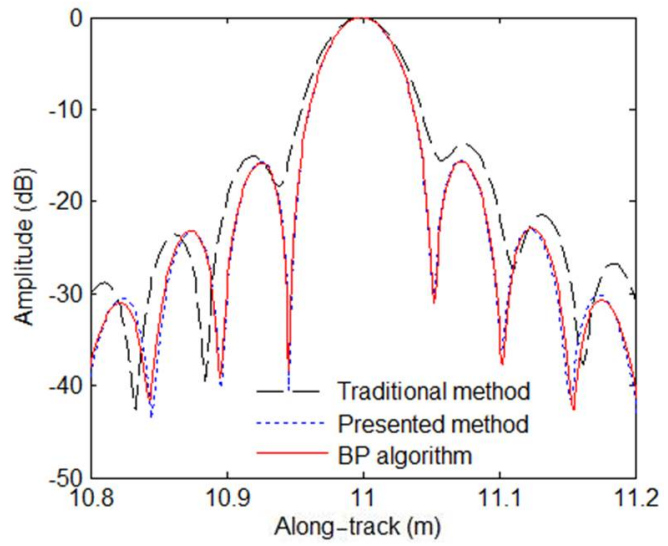

(b)

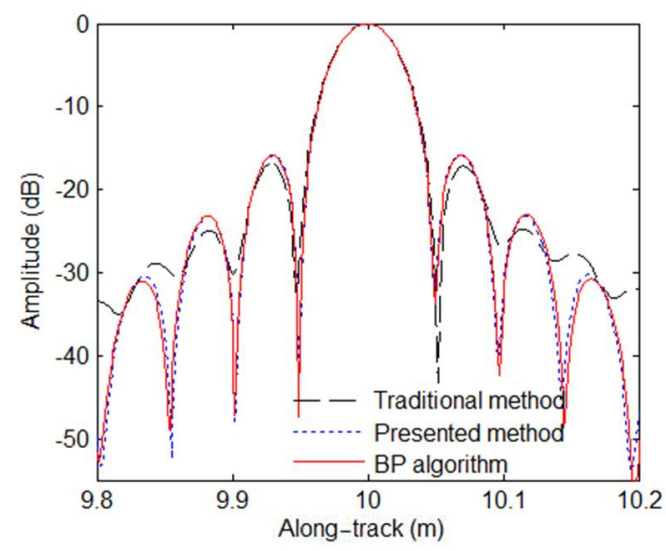

(c)

Figure 17. Along-track slices with the case of a wide-bandwidth signal. (a) T1; (b) T2; (c) T3.

Quality parameters including along-track resolution, PLSR, and ISLR are listed in Table 6. Inspecting quality parameters of T1, the PSLR and ISLR of the traditional method [38] significantly deviate from corresponding values of the BP algorithm by $2.54 \mathrm{~dB}$ and $3.71 \mathrm{~dB}$. Therefore, the along-track resolution is broadened. The differences of PSLR and ISLR between the presented method and $\mathrm{BP}$ algorithm are just $0.2 \mathrm{~dB}$ and $0.01 \mathrm{~dB}$. Based on the quality parameters of T2 and T3, the imaging performance of the presented method is still superior to that of the traditional method.

Table 6. Comparison of imaging quality with the wide-bandwidth case.

\begin{tabular}{ccccc}
\hline Method & Resolution $(\mathbf{m})$ & PLSR $(\mathbf{d B})$ & ISLR $(\mathbf{d B})$ & Target \\
\hline Traditional method & 0.05 & -13.31 & -5.26 & \\
Presented method & 0.04 & -15.65 & -8.96 & $\mathrm{~T} 1$ \\
BP algorithm & 0.04 & -15.85 & -8.97 & \\
\hline Traditional method & 0.05 & -13.82 & -6.86 & \\
Presented method & 0.04 & -15.61 & -9.61 & $\mathrm{~T} 2$ \\
BP algorithm & 0.04 & -15.66 & -9.65 & \\
\hline Traditional method & 0.04 & -16.18 & -10.62 & \\
Presented method & 0.04 & -15.79 & -10.83 & $\mathrm{~T} 3$ \\
BP algorithm & 0.04 & -15.83 & -10.91 & \\
\hline
\end{tabular}

Based on the simulations discussed above, we conclude that the signal bandwidth highly affects the imaging performance of the traditional R-D algorithm [38]. The traditional R-D algorithm [38] is just suitable for the SAS imagery when the narrow-bandwidth signal is used. However, the defocusing would occur, as large PTRS error and residual quadratic 
coupling error are introduced when the SAS system works with a wide-bandwidth signal. With the presented method, the targets can be well recovered with both cases, and the processing results are mostly close to results based on the BP algorithm.

\subsection{Real Data Processing}

\subsubsection{Results with the Narrow-Bandwidth Case}

In this section, real data is exploited to further validate the presented method. Firstly, the multi-receiver SAS data with a narrow-bandwidth signal are used to test the presented method. The parameters are shown in Table 7.

Table 7. Real SAS parameters with a narrow-bandwidth signal.

\begin{tabular}{ccc}
\hline Parameter & Value & Unit \\
\hline Platform velocity & 2.5 & $\mathrm{~m} / \mathrm{s}$ \\
Pulse repetition interval & 0.32 & $\mathrm{~s}$ \\
Signal bandwidth & 20 & $\mathrm{kHz}$ \\
Carrier frequency & 150 & $\mathrm{kHz}$ \\
Receiver array length & 1.2 & $\mathrm{~m}$ \\
Receiver element width & 0.04 & $\mathrm{~m}$ \\
Transmitter width & 0.08 & $\mathrm{~m}$ \\
\hline
\end{tabular}

The traditional R-D algorithm, presented method, and BP algorithm are used to process the real data. The resultant images are shown in Figure 18. The images at the top left in Figure 18 are the close-looking results of the target marked by a red circle in Figure 18a. Compared to Figure 18c, the image based on the traditional R-D algorithm can still process the echoed signal without loss of performance. That is to say, the traditional R-D algorithm is suitable for SAS imagery when the narrow-bandwidth signal is used. With the presented method, the image shown in Figure 18b agrees well with that of the BP algorithm. In other words, the presented method can well focus targets with the case of a narrow-bandwidth signal.
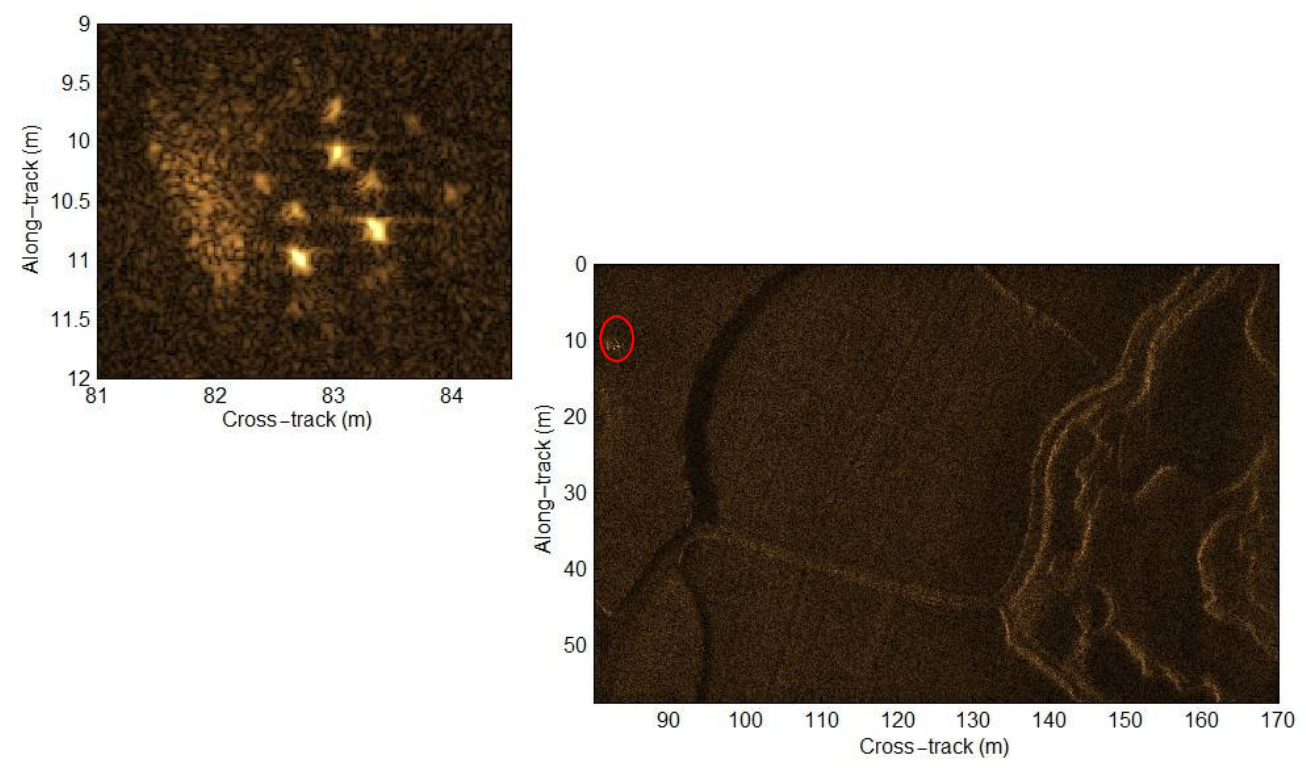

(a)

Figure 18. Cont. 

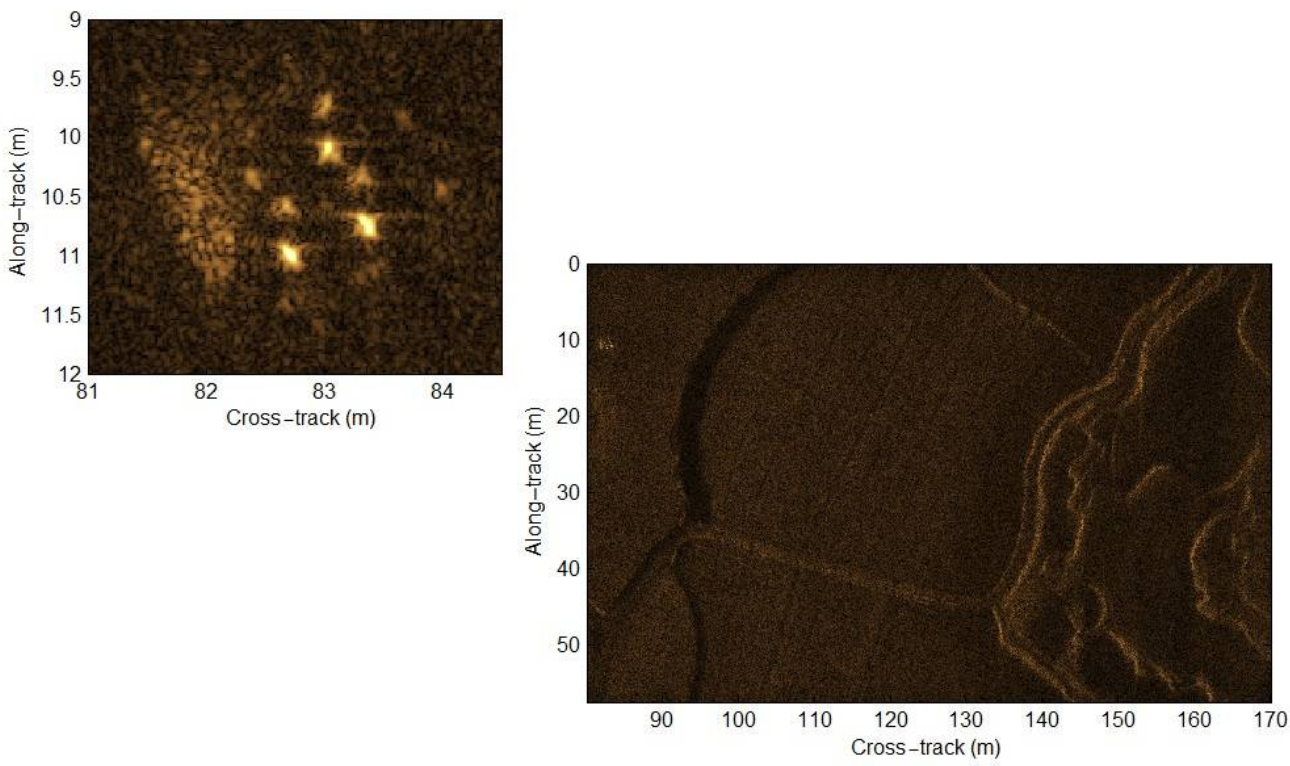

(b)
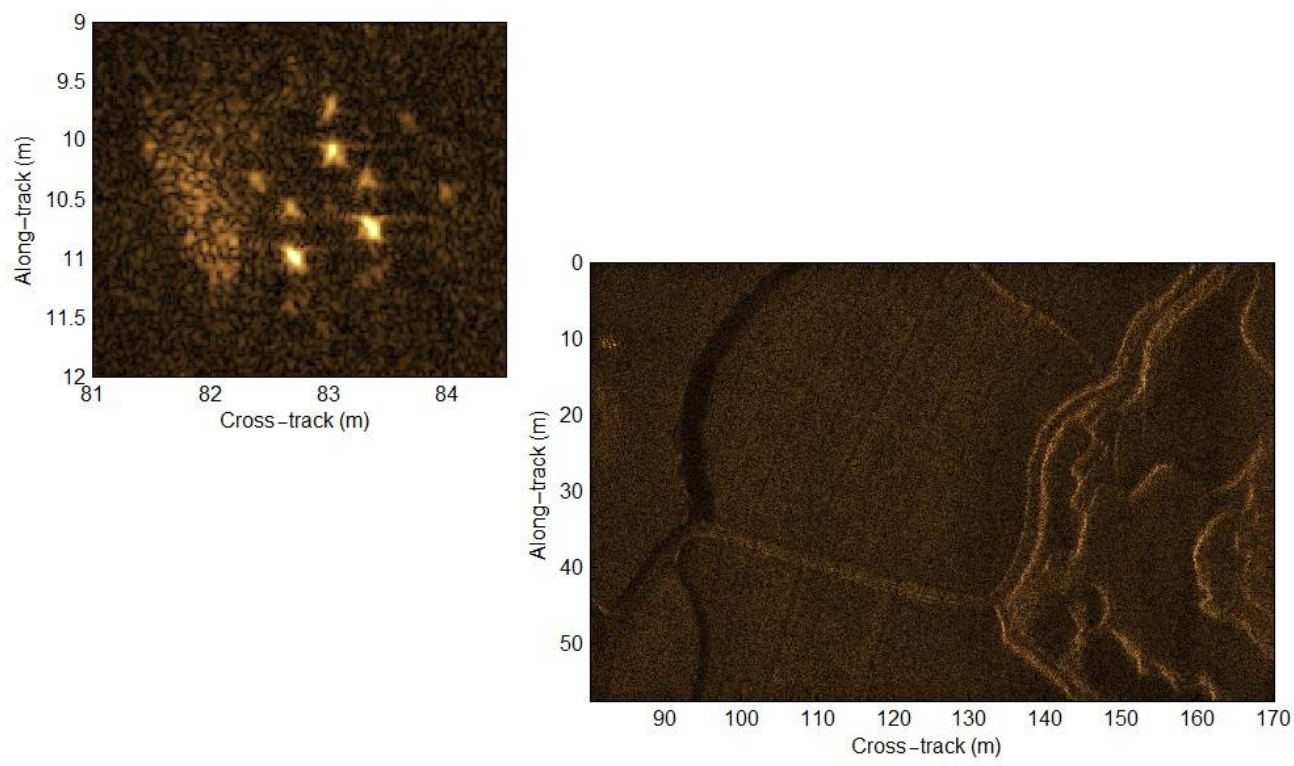

(c)

Figure 18. Real data processing results with the case of a narrow-bandwidth signal. (a) Traditional method; (b) presented method; (c) BP algorithm.

The along-track slices related to the target marked by a red circle in Figure 18a are shown in Figure 19. From Figure 19, we find that the slices of the traditional method and presented method agree well with the curve of the BP algorithm. Based on Figure 19, the resolution after imagery with the $\mathrm{BP}$ algorithm is $0.090 \mathrm{~m}$. The resolution of the presented method is $0.091 \mathrm{~m}$, while that of the traditional method is $0.095 \mathrm{~m}$. It can be found that the traditional method can get high-resolution results without a loss of imaging performance. This indicates that the traditional method and presented method are suitable for SAS imagery when the narrow-bandwidth signal is exploited. 


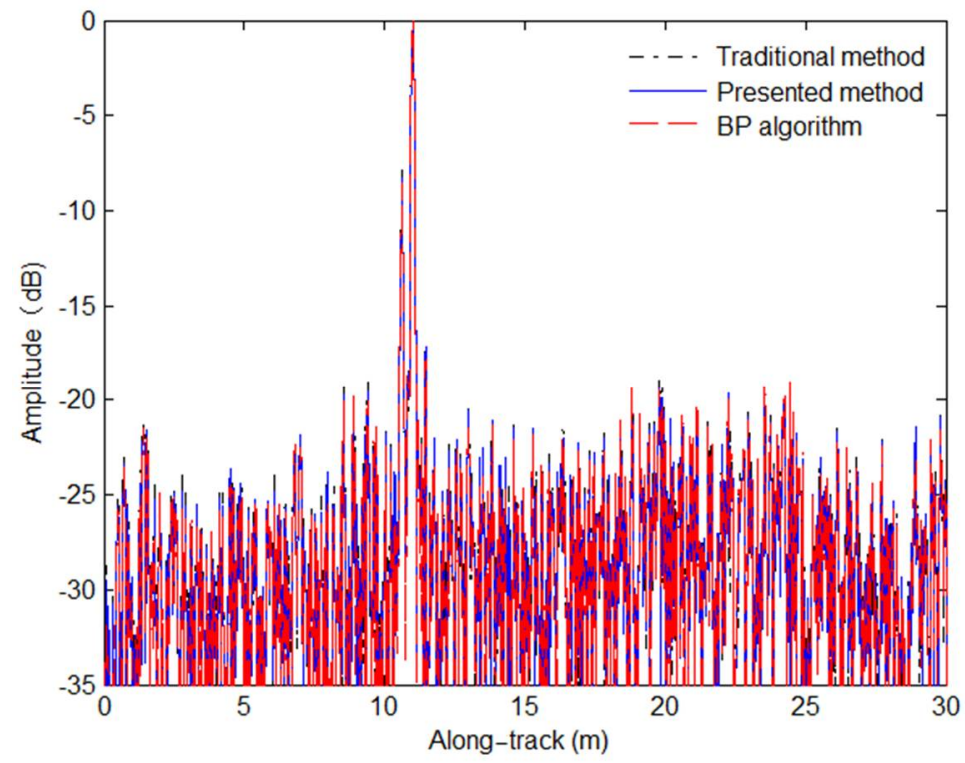

(a)

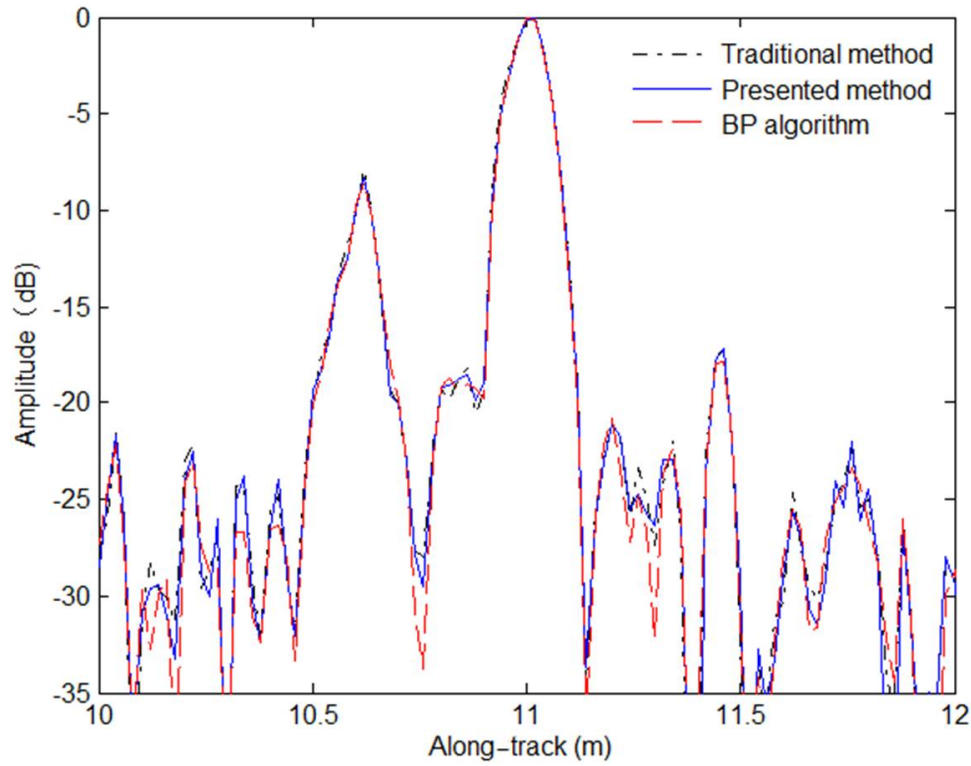

(b)

Figure 19. Cross-track slices of a target marked by a red circle in (a). (a) Global slices; (b) close look.

5.2.2. Results with a Wide-Bandwidth Case

Here, the real SAS data based on a wide-bandwidth signal are further used to validate the presented method. The SAS parameters with a wide-bandwidth signal are listed in Table 8 . It can be found that the SAS system works with a wide-bandwidth signal.

Table 8. Real SAS parameters with a wide-bandwidth signal.

\begin{tabular}{ccc}
\hline Parameter & Value & Unit \\
\hline Platform velocity & 2 & $\mathrm{~m} / \mathrm{s}$ \\
Pulse repetition interval & 0.06 & $\mathrm{~s}$ \\
Signal bandwidth & 7 & $\mathrm{kHz}$ \\
Carrier frequency & 12 & $\mathrm{kHz}$ \\
Receiver array length & 0.24 & $\mathrm{~m}$ \\
Receiver element width & 0.08 & $\mathrm{~m}$ \\
Transmitter width & 0.08 & $\mathrm{~m}$ \\
\hline
\end{tabular}


The real data is processed by using the traditional R-D algorithm [38], presented method, and BP algorithm [42]. The resultant images are shown in Figure 20. The images at the top left in Figure 20 are the close-looking results of the target marked by a red circle in Figure 20a. It can be clearly found that the result of the traditional method in Figure 20a suffers from some distortion compared to that of the BP algorithm shown in Figure 20c. However, the presented method can still provide a high-performance result, which is close to that of the BP algorithm.
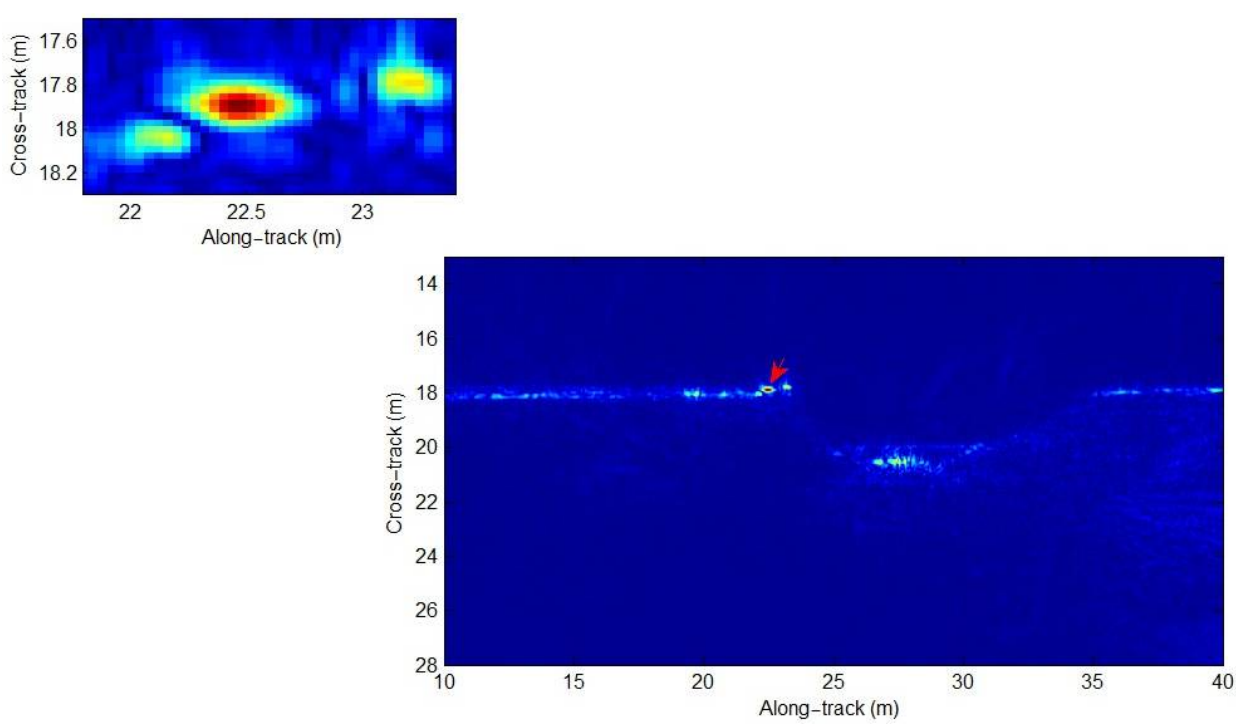

(a)
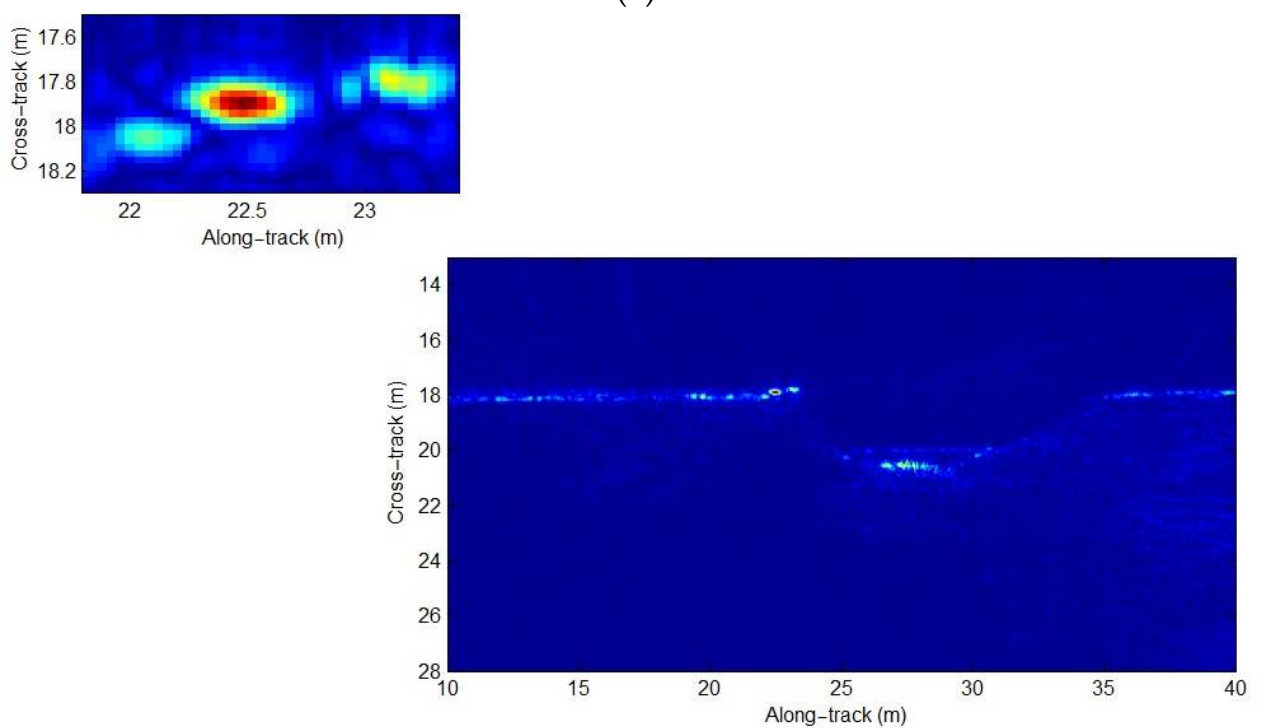

(b)

Figure 20. Cont. 

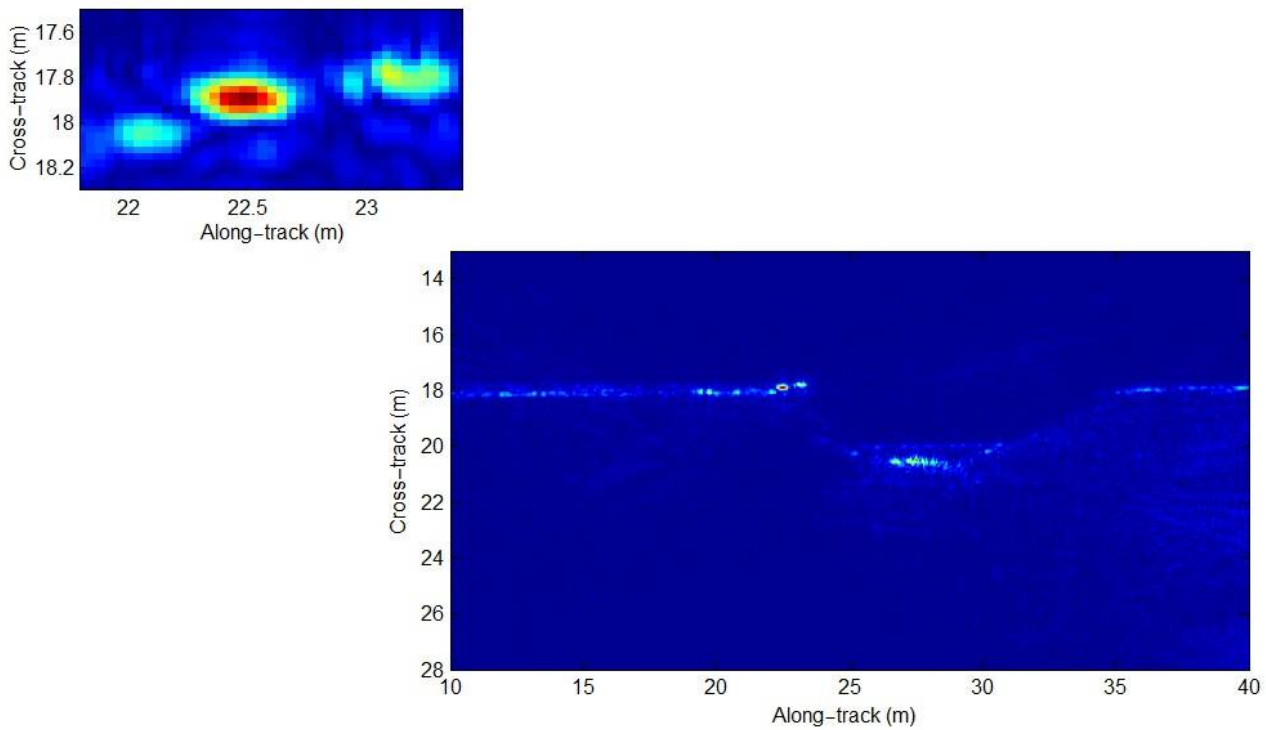

(c)

Figure 20. Real data processing results with the case of a wide-bandwidth signal. (a) Traditional method; (b) presented method; (c) BP algorithm.

We focus on the target marked by a red arrow in Figure 20a. The along-track slices are depicted in Figure 21 to visually compare the imaging performance. From Figure 21, we clearly find that the curve of the traditional method [38] deviates from that of the BP algorithm [42]. Therefore, the imaging performance of the traditional method is lowered when the wide-bandwidth signal is exploited. Based on Figure 21, the resolution of the BP algorithm is $0.252 \mathrm{~m}$. The resolution of the presented method is $0.257 \mathrm{~m}$, while that of the traditional method is $0.291 \mathrm{~m}$. The resolution difference between the traditional method and the BP algorithm is $0.039 \mathrm{~m}$. Fortunately, the slice of the presented method is mostly close to that of the BP algorithm [42], and the resolution difference between the presented method and the BP algorithm is $0.005 \mathrm{~m}$. This indicates that the presented method can obtain high-performance results with the wide-bandwidth case. This conclusion agrees well with that drawn from Section 5.1.

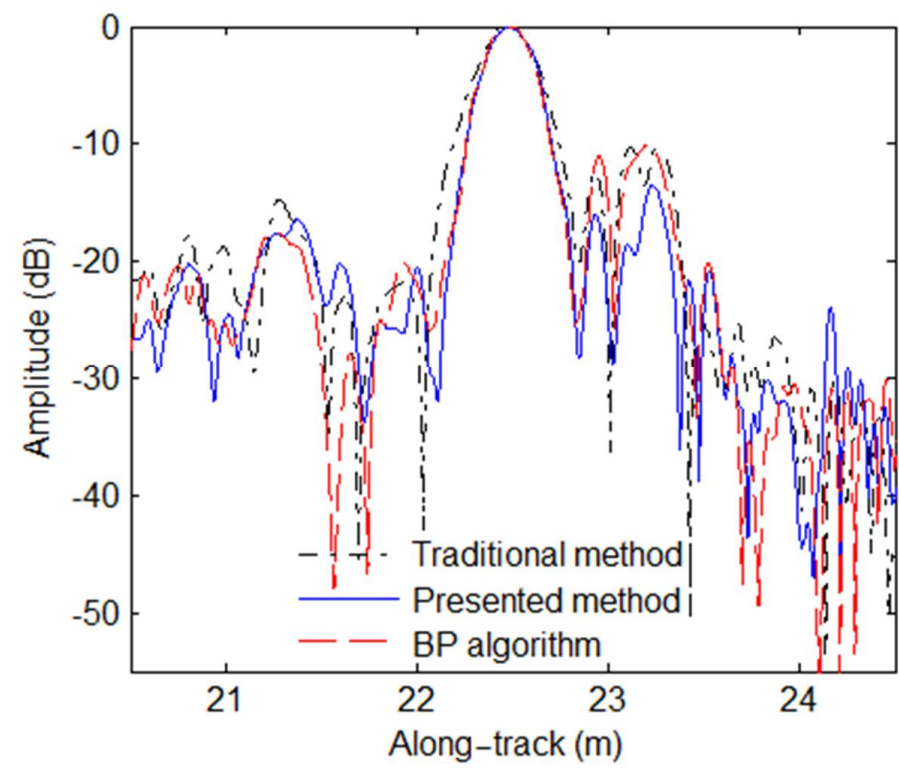

Figure 21. Along-track slices of target marked by a red arrow in Figure 20a. 


\section{Discussion}

In general, the narrowband signal and wideband signal are relative terms. To simplify the processing, the narrowband signal model is often used. The center frequency $f_{c}$ is large compared to the signal bandwidth $B$, and this signal can be considered to be the narrowband signal. With a mathematical expression, the signal would be a narrowband signal when an inequality $B \ll f_{c}$ is satisfied. In engineering applications, the signal can be considered to be the narrow bandwidth signal if the inequality $B / f_{c}<0.1$ holds. Considering this condition, many SAS systems are characterized by a wideband, which is also drawn by Pailhas in [35]. Therefore, traditional imaging methods cannot be directly applied to these SAS systems, and traditional imaging methods should be extended.

In this paper, we mainly focus on the extension of the traditional R-D algorithm. The approximation errors of the multi-receiver SAS R-D algorithm are first analyzed. With our analysis, the approximation errors can be negligible when the narrow-bandwidth signal is used by an SAS system. That is to say, the imaging performance is nearly unaffected by the approximation errors. Unfortunately, the approximation errors are large when the SAS system works with a wide-bandwidth signal. In other words, the residual phase error would seriously distort the imaging performance in the wide-bandwidth case. Based on simulations and real data processing results, the traditional R-D algorithm can only obtain high-resolution images in the narrow-bandwidth case. When it comes to the SAS imagery with a wide-bandwidth signal, the results based on the traditional R-D algorithm are seriously distorted. These conclusions are completely consistent with conclusions drawn from the analysis of approximation errors. With the presented method in this paper, the data in the case of a narrow-bandwidth signal can be well processed. In general, there is a slight difference in processing performance between the traditional method and presented method when the narrow-bandwidth signal is used. Since the imaging performance of the presented method is still similar to that of the $\mathrm{BP}$ algorithm, the major difference in imaging performance between the traditional method and presented method is caused in the case of a wide-bandwidth signal. Summarily, the approximation errors neglected by traditional imaging algorithms are compensated by the presented method based on the sub-block processing method in the cross-track dimension. At this point, the targets across the whole swath can be well focused.

\section{Conclusions}

From Sections 3-5, we find that the presented method can obtain a well-focused image in the case of a wide-bandwidth signal. The main factors influencing the multi-receiver SAS imagery with a wide-bandwidth signal include PTRS error and residual quadratic coupling error. Both errors are compensated by using the sub-block processing method in the cross-track dimension. With the presented method, we can obtain a better focusing performance than with the traditional method.

It should be noted that the sub-block processing method lowers the imaging efficiency, as the compensation of approximation errors should be repeatedly conducted for each sub-block. This is the major limitation of the presented method. Fortunately, parallel algorithms and graphics processing units (GPU) can be exploited to improve the efficiency to some degree. The real data often suffer from motion error. In this paper, we do not consider the compensation of motion error for real data processing. It is expected that the navigation data and autofocus techniques are exploited to further improve the focusing performance of real data, which is our future work.

Author Contributions: Conceptualization, X.Z.; methodology, X.Z.; software, X.Z.; validation, X.Z. and P.Y.; formal analysis, P.Y.; investigation, P.Y.; resources, P.Y.; data curation, P.Y.; writing—original draft preparation, X.Z.; writing-review and editing, X.Z.; visualization, P.Y.; supervision, X.Z.; project administration, X.Z.; funding acquisition, X.Z. All authors have read and agreed to the published version of the manuscript. 
Funding: This research was funded by National Key Laboratory Foundation under grant No. 9140C290401150C29132.

Institutional Review Board Statement: Not applicable.

Informed Consent Statement: Not applicable.

Data Availability Statement: Not applicable.

Acknowledgments: The authors would like to thank the editor and anonymous reviewers for their helpful comments and suggestions that have helped enhance the quality of the manuscript. Moreover, we are very grateful for loved Yuchen Zhangyang, who gives us strength to continuously move forward.

Conflicts of Interest: The authors declare no conflict of interest.

\section{References}

1. Maurya, H.; Kumar, A.; Mishra, A.K.; Panigrahi, R.K. Improved four-component based polarimetric synthetic aperture radar image decomposition. IET RadarSonar Navig. 2020, 14, 619-627. [CrossRef]

2. Alves, D.I.; Palm, B.G.; Hellsten, H.; Vu, V.T.; Pettersson, M.I.; Machado, R.; Uchôa-Filho, B.F.; Dammert, P. Wavelength-resolution SAR change detection using Bayes' theorem. IEEE J. Sel. Top. Appl. Earth Obs. Remote Sens. 2020, 13, 5560-5568. [CrossRef]

3. Hashempour, H.R. Fast ADMM-based approach for high-resolution ISAR imaging. Electron. Lett. 2020, 56, 954-957. [CrossRef]

4. Wijayasiri, A.; Banerjee, T.; Ranka, S.; Sahni, S.; Schmalz, M. Multiobjective optimization of SAR reconstruction on hybrid multicore systems. IEEE J. Sel. Top. Appl. Earth Obs. Remote Sens. 2020, 13, 4674-4688. [CrossRef]

5. Corbett, B.; Andre, D.; Finnis, M. Localising vibrating scatterer phenomena in synthetic aperture radar imagery. Electron. Lett. 2020, 56, 395-398. [CrossRef]

6. Pirrone, D.; Bovolo, F.; Bruzzone, L. An approach to unsupervised detection of fully and partially destroyed buildings in multitemporal VHR SAR images. IEEE J. Sel. Top. Appl. Earth Obs. Remote Sens. 2020, 13, 5938-5953. [CrossRef]

7. Otsuka, Y.; Shimada, T.; Natsuaki, R.; Hirose, A. Consideration on singular-point generating mechanisms by analyzing the effect of phase-and-polarization optimization in PolInSAR. IEEE J. Sel. Top. Appl. Earth Obs. Remote Sens. 2020, 13, 1625-1638. [CrossRef]

8. Saqueb, S.; Nahar, N.K.; Sertel, K. Fast two-dimensional THz imaging using rail-based synthetic aperture radar (SAR) processing. Electron. Lett. 2020, 56, 988-990. [CrossRef]

9. AlShaya, M.; Yaghoobi, M.; Mulgrew, B. Ultrahigh resolution wide swath MIMO-SAR. IEEE J. Sel. Top. Appl. Earth Obs. Remote Sens. 2020, 13, 5358-5368. [CrossRef]

10. Nithirochananont, U.; Antoniou, M.; Chemiakov, M. Passive coherent multistatic SAR using spaceborne illuminators. IET Radar Sonar Navig. 2020, 14, 628-636. [CrossRef]

11. Brown, D.; Cook, D.A.; Fernandez, J. Results from a small synthetic aperture sonar. In Proceedings of the MTS/IEEE Oceans Conference, Boston, MA, USA, 18-22 September 2006; pp. 1-6.

12. Campbell, J.D.; Chang, E. Concepts for synthetic aperture sonar performance prediction and mission planning. In Proceedings of the MTS/IEEE Oceans Conference, Washington, DC, USA, 17-23 September 2005; pp. 22-29.

13. Billon, D.; Pinto, M. Some general considerations for synthetic aperture sonar design. In Proceedings of the MTS/IEEE Oceans Conference, San Diego, CA, USA, 9-12 October 1995; pp. 1665-1670.

14. Cuenca-García, C.; Risbøl, O.; Bates, C.R.; Stamnes, A.A.; Skoglund, F.; Ødegård, Ø.; Viberg, A.; Koivisto, S.; Fuglsang, M.; Gabler, M.; et al. Sensing archaeology in the north: The use of non-destructive geophysical and remote sensing methods in archaeology in scandinavian and north atlantic territories. Remote Sens. 2020, 12, 3102. [CrossRef]

15. Zwolak, K.; Wigley, R.; Bohan, A.; Zarayskaya, Y.; Bazhenova, E.; Dorshow, W.; Sumiyoshi, M.; Sattiabaruth, S.; Roperez, J.; Proctor, A.; et al. The autonomous underwater vehicle integrated with the unmanned surface vessel mapping the southern Ionian sea. the winning technology solution of the shell ocean discovery XPRIZE. Remote Sens. 2020, 12, 1344. [CrossRef]

16. Zhang, X.; Yang, P. Imaging algorithm for multireceiver synthetic aperture sonar. J. Electr. Eng. Technol. 2019, 14, 471-478. [CrossRef]

17. Steele, S.; Charron, R.; Dillon, J.; Shea, D. Shallow water survey with a miniature synthetic aperture sonar. In Proceedings of the MTS/IEEE Oceans Conference, Seattle, WA, USA, 27-31 October 2019; pp. 1-6.

18. Ånonsen, K.B.; Hagen, O.K.; Berglund, E. Autonomous mapping with AUVs using relative terrain navigation. In Proceedings of the MTS/IEEE Oceans Conference, Anchorage, AK, USA, 18-21 September 2017; pp. 1-7.

19. Tellez, O.L. Underwater threat recognition: Are automatic target classification algorithms going to replace expert human operators in the near future? In Proceedings of the MTS/IEEE Oceans Conference, Marseille, France, 17-20 June 2019; pp. 1-4.

20. Chhabra, P.S.; Pailhas, Y.; Wallace, A.M.; Hopgood, J.R.; Petíllot, Y.R. Target classification in SAS imagery using orthogonal basis selection. In Proceedings of the MTS/IEEE Oceans Conference, Aberdeen, UK, 19-22 June 2017; pp. 1-10.

21. LeHardy, P.K.; Larsen, J. Deepwater synthetic aperture sonar and the search for MH370. In Proceedings of the MTS/IEEE Oceans Conference, Washington, DC, USA, 19-22 October 2015; pp. 1-4.

22. Abu, A.; Diamant, R. CFAR detection algorithm for objects in sonar images. IET Radar Sonar Navig. 2020, 14, 1757-1766. [CrossRef] 
23. Shannon, C.E. Communication in the presence of noise. Proc. IEEE 1984, 72, 1192-1201. [CrossRef]

24. Callow, H.J. Signal Processing for Synthetic Aperture Sonar Image Enhancement; University of Canterbury: Christchurch, New Zealand, 2003.

25. Sawa, T.; Kasaya, T.; Nakatsuka, K.; Tanaka, N.; Furushima, Y. Improvement of synthetic aperture sonar with multi-channel projector. In Proceedings of the MTS/IEEE Oceans Conference, Washington, DC, USA, 19-22 October 2015; pp. 1-6.

26. Zhang, X.; Tan, C.; Ying, W. An imaging algorithm for multireceiver synthetic aperture sonar. Remote Sens. 2019, 11, 672. [CrossRef]

27. Long, T.; Hu, C.; Ding, Z.; Dong, X.; Tian, W.; Zeng, T. Geosynchronous SAR: System and Signal Processing; Springer Nature: Singapore, 2018.

28. Xu, W.; Sun, F.; Fan, S.; Pan, X. A unified framework for multiple-input multiple-output and bistatic synthetic aperture sonar processing. In Proceedings of the MTS/IEEE Oceans Conference, Genova, Italy, 18-21 May 2015; pp. 1-4.

29. LePage, K.D.; Schmidt, H. Bistatic synthetic aperture imaging of proud and buried targets from an AUV. IEEE J. Ocean. Eng. 2002, 27, 471-483. [CrossRef]

30. Loffeld, O.; Nies, H.; Peters, V.; Knedlik, S. Models and useful relations for bistatic SAR processing. IEEE Trans. Geosci. Remote Sens. 2004, 42, 2031-2038. [CrossRef]

31. Gough, P.T.; Hayes, M.P.; Wilkinson, D.R. An efficient image reconstruction algorithm for a muliple hydrophone array synthetic aperture sonar. In Proceedings of the 5th European Conference on Underwater Acoustics (ECUA2000), Lyon, France, 10-13 July 2000; pp. 395-400.

32. Bonifant, W.W.; Richards, M.; McClellan, J. Interferometric height estimation of the seafloor via synthetic aperture sonar in the presence of motion errors. IEE Proc. RadarSonar Navig. 2000, 147, 322-330. [CrossRef]

33. Bellettini, A.; Pinto, M.A. Theoretical accuracy of synthetic aperture sonar micronavigation using a displaced phase-center antenna. IEEE J. Ocean. Eng. 2002, 27, 780-789. [CrossRef]

34. Zhang, X.; Tang, J.; Zhong, H. Multireceiver correction for the chirp scaling algorithm in synthetic aperture sonar. IEEE J. Ocean. Eng. 2014, 39, 472-481. [CrossRef]

35. Pailhas, Y.; Dugelay, S.; Capus, C. Impact of temporal Doppler on synthetic aperture sonar imagery. J. Acoust. Soc. Am. 2018, 143, 318-329. [CrossRef] [PubMed]

36. Zhang, X.; Liu, Y.; Deng, X. Influence of phase centre approximation error on SAS imagery. In Proceedings of the 2021 IEEE the 6th International Conference on Computer and Communication Systems (ICCCS 2021), Chengdu, China, 23-26 July 2021; pp. 352-356.

37. Zhang, X.; Chen, X.; Qu, W. Influence of the stop-and-hop assumption on synthetic aperture sonar imagery. In Proceedings of the 2017 17th IEEE International Conference on Communication Technology (ICCT 2017), Chengdu, China, 27-30 October 2017; pp. 1601-1607.

38. Lv, J.; Tang, F.; Zhao, X.; Wu, H. A small squint range doppler algorithm for the multi-receiver synthetic aperture sonar. Ship Sci. Technol. 2019, 41, 124-129.

39. Cumming, I.; Bennett, J.R. Digital processing of Seasat SAR data. In Proceedings of the IEEE International Conference on Acoustics, Speech, and Signal Processing (ICASSP), Washingto, DC, USA, 12-15 May 1998; pp. 710-718.

40. Chang, C.Y.; Jin, M.; Curlander, J.C. Squint mode SAR processing algorithms. In Proceedings of the IEEE International Geoscience and Remote Sensing Symposium (IGARSS), Vancouver, BC, Canada, 24-29 July 2021; pp. 1702-1706.

41. Jin, M.J.; Wu, C. A SAR correlation algorithm which accommodates large range migration. IEEE Trans. Geosci. Remote Sens. 1984, 22, 592-597. [CrossRef]

42. Zhang, X.; Yang, P.; Ying, W. BP algorithm for the multireceiver SAS system. IET Radar Sonar Navig. 2019, 13, 830-838. [CrossRef]

43. Duersch, M.I. Backprojection for Synthetic Aperture Radar; Brigham Young University: Provo, UT, USA, 2013.

44. Ødegaard, N. Fast Time Domain Beamforming for Synthetic Aperture Sonar; University of Oslo: Oslo, Norway, 2004.

45. Johnson, K.A.; Hayes, M.P.; Gough, P.T. A method for estimating the sub-wavelength sway of a sonar towfish. IEEE J. Ocean. Eng. 1995, 20, 258267. [CrossRef] 